Cahiers québécois de démographie

Revue internationale d'étude des populations

\title{
Le vieillissement de la population et la décroissance démographique en milieu rural : un défi pour le développement territorial des petites localités du Québec Aging and population decline in rural areas: a challenge to the territorial development of small rural communities
}

\section{Majella Simard}

Volume 48, numéro 2, automne 2019

URI : https://id.erudit.org/iderudit/1074178ar

DOI : https://doi.org/10.7202/1074178ar

Aller au sommaire du numéro

Éditeur(s)

Association des démographes du Québec

ISSN

1705-1495 (numérique)

Découvrir la revue

Citer cet article

Simard, M. (2019). Le vieillissement de la population et la décroissance démographique en milieu rural : un défi pour le développement territorial des petites localités du Québec. Cahiers québécois de démographie, 48(2), 107-134. https://doi.org/10.7202/1074178ar
Résumé de l'article

Le Québec est la troisième province qui a été la plus affectée par le vieillissement de sa population entre 1986 et 2016. En outre, le phénomène tend à s'accentuer avec la réduction de la taille démographique des localités, si bien que les plus petites apparaissent les plus concernées par cette réalité qui se voit amplifiée par la diminution de la proportion des moins de 24 ans. Le but de cet article est d'identifier les petites localités rurales nécessitant une intervention au moyen d'une typologie construite à partir de diverses caractéristiques démographiques. Cette typologie permettra, par la suite, de proposer quelques pistes en vue de favoriser un aménagement plus harmonieux du territoire. Le modèle de développement territorial intégré sert de cadre théorique à l'analyse. Les résultats reflètent une situation inquiétante notamment au sein de 81 petites localités qui réclament des actions en vue de redresser leur démographie et de favoriser une meilleure occupation du territoire.
Tous droits réservés (C) Association des démographes du Québec, 2021

Ce document est protégé par la loi sur le droit d'auteur. L'utilisation des services d'Érudit (y compris la reproduction) est assujettie à sa politique d'utilisation que vous pouvez consulter en ligne.

https://apropos.erudit.org/fr/usagers/politique-dutilisation/ 


\title{
Cahiers québécois de démographie
} Vol. 48, n² 2, automne 2019, p. 107-134

\section{Le vieillissement de la population et la décroissance démographique en milieu rural: un défi pour le développement territorial des petites localités du Québec}

\author{
MAJELLA SIMARD ${ }^{*}$
}

\begin{abstract}
RÉSUMÉ
Le Québec est la troisième province qui a été la plus affectée par le vieillissement de sa population entre 1986 et 2016. En outre, le phénomène tend à s'accentuer avec la réduction de la taille démographique des localités, si bien que les plus petites apparaissent les plus concernées par cette réalité qui se voit amplifiée par la diminution de la proportion des moins de 24 ans. Le but de cet article est d'identifier les petites localités rurales nécessitant une intervention au moyen d'une typologie construite à partir de diverses caractéristiques démographiques. Cette typologie permettra, par la suite, de proposer quelques pistes en vue de favoriser un aménagement plus harmonieux du territoire. Le modèle de développement territorial intégré sert de cadre théorique à l'analyse. Les résultats reflètent une situation inquiétante notamment au sein de 81 petites localités qui réclament des actions en vue de redresser leur démographie et de favoriser une meilleure occupation du territoire.
\end{abstract}

\begin{abstract}
Aging and population decline in rural areas: a challenge to the territorial development of small rural communities

Quebec is the third most affected province in Canada in terms of aging of the population between 1986 and 2016. Moreover, the phenomenon tends to be accentuated with the depopulation of some localities, the smallest being the most concerned by this phenomenon, and particularly by the decrease in the proportion of those under age 25 . The purpose of this article is to identify small rural communities requiring intervention, using a typology constructed from demographic characteristics. This typology will enable, as a second step, the proposal of ways to promote a more harmonious management of the territory. The territorial development model serves as a theoretical framework for the analysis. The results reflect a worrisome situation, particularly in 81 small towns that are calling for action to improve their demographic profiles and promote a better use of the land.
\end{abstract}

* Ph. D., Département d'histoire et de géographie, Université de Moncton, majella.simard@umoncton.ca 


\section{INTRODUCTION}

T e vieillissement de la population, défini par l'augmentation de la proportion des personnes âgées de 65 ans et plus au cours d'une période donnée, représente l'un des enjeux fondamentaux du présent siècle (Organisation des Nations unies, 2017). Selon la Déclaration politique et le Plan d'action international de Madrid sur le vieillissement, dont le Canada est l'un des cosignataires, il s'agit d'un phénomène sans précédent, universel, de grande portée, durable et irréversible, dont l'évaluation des manifestations et des impacts reste complexe (Organisation des Nations unies, 2003). La montée en âge des générations relativement nombreuses nées lors du renouveau démographique suivant la Seconde Guerre mondiale, conjuguée à l'allongement de l'espérance de vie et à une natalité affaiblie, a effectivement pour effet de favoriser une instabilité croissante des structures d'âge. À ces facteurs s'ajoutent le jeu des mouvements migratoires qui, à certains endroits, peut contribuer à amplifier ce déséquilibre, et celui de l'héritage démographique caractérisé par "l'arrivée à l'âge de la retraite des générations plus nombreuses depuis 2005 » (Dumont, 2016: 57).

Après Terre-Neuve-et-Labrador $(10,6)^{1}$ et le Nouveau-Brunswick $(8,8)$, le Québec $(8,3)$ est la province canadienne qui, entre 1986 et 2016, a été la plus touchée par le vieillissement de sa population (Statistique Canada, 2016; 1986). En braquant les projecteurs sur un échelon territorial plus fin, le vieillissement apparaît comme un phénomène bien présent au sein des localités rurales du Québec. De 1986 à 2016, la proportion d'aînés s'est accrue de 11,1 points de pourcentage, comparativement à 8,3 pour l'ensemble de la province. En outre, le vieillissement tend à s'accentuer avec la réduction de la taille démographique des localités, les plus petites étant particulièrement concernées par ce phénomène. Ainsi, l'accroissement de la proportion d'aînés entre 1986 et 2016 a atteint 12,2 points au sein des petites localités rurales du Québec contre 9,3 pour les municipalités dont le nombre d'habitants varie entre 2 ooo et 2499.

Eu égard à un tel constat, il est indéniable que le vieillissement comporte des enjeux et des défis particuliers au sein des petites localités rurales du Québec en matière d'aménagement et de développement territorial, mais aussi en ce qui a trait aux changements concernant les besoins des aînés qui vivent au sein de ces petits milieux que ce soit sur le plan du logement, des services sociaux, de la santé, des transports ou des loisirs (Lord et Piché, 2018; Simard, 2015b). C'est dans cet esprit que s'inscrit cet article, dont l'objectif consiste à identifier, au moyen d'une typologie construite à partir de diverses caractéristiques démographiques, les petites localités rurales les plus enclines au vieillissement et à la décroissance. L'atteinte de cet objectif passe en premier lieu par un bref examen des trajectoires évolutives du nombre et de la proportion de personnes âgées et de jeunes au sein des petites localités rurales du Québec. Cet examen apparaît incontournable afin d'identifier les mesures qui pourraient être mises de l'avant dans le but de favoriser un développement plus équilibré et une occupation plus harmonieuse du territoire.

Cet article comporte cinq parties. Dans la première sont exposés les principaux éléments du cadre conceptuel. La seconde dresse l'état des lieux du vieillissement au sein des petites localités

1. Le chiffre entre parenthèses indique l'augmentation de la proportion des personnes âgées de 65 ans et plus entre 1986 et 2016. Il est exprimé en points de pourcentage. Précisons que toutes les données utilisées dans le cadre de cet article sont issues des recensements de Statistique Canada. 
rurales du Québec. La troisième partie présente la méthode d'analyse retenue eu égard à la présentation des données. La quatrième porte sur l'analyse des résultats proprement dits. Enfin, dans la cinquième, quelques mesures susceptibles d'atténuer les effets du vieillissement et de la décroissance démographique sont examinées, ceci dans le but de promouvoir un aménagement plus harmonieux du territoire.

\section{CADRE CONCEPTUEL}

D’entrée de jeu, il importe de préciser ce qui est entendu par «localité », " petite localité » et « localité rurale». Dans le cadre de cet article, la localité renvoie à la subdivision de recensement de Statistique Canada, c'est-à-dire au « terme générique qui désigne les municipalités (telles que définies par les lois provinciales/territoriales) ou les territoires considérés comme étant des équivalents municipaux à des fins statistiques (p. ex. : les réserves indiennes, les établissements indiens et les territoires non organisés) »(Statistique Canada, 2016). Quant aux petites localités, il s'agit du segment de l'espace rural formé des 202 subdivisions de recensement dont la population était inférieure à 500 habitants en 2016. Ces dernières constituent près de $20 \%$ de l'espace rural québécois. Enfin, l'expression « localité rurale » désigne les subdivisions dont la taille démographique est inférieure à 2500 habitants. Au dernier recensement de 2016, celles-ci étaient au nombre de 719 .

Dans ses travaux, Gérard-François Dumont insiste sur la nécessité de distinguer les notions de « vieillissement » et de " gérontocroissance », les deux phénomènes divergeant à la fois dans leur définition, leurs facteurs d'intensité et leurs prospectives démographiques. Le vieillissement est la résultante de trois processus : l'accroissement du nombre de personnes âgées (attribuable à l'augmentation de l'espérance de vie, mais aussi à l'arrivée des baby-boomers parmi les 65 ans et plus), la dénatalité (qui entraîne un vieillissement "par le bas »)2 et les courants migratoires (qui se manifestent soit par un déficit ou par un excédent occasionnant, du coup, le vieillissement de la population). Dès lors, le vieillissement crée un effet de structure, c'est-à-dire une transformation dans la composition des pyramides d'âge (Dumont, 2016). La gérontocroissance réfère, quant à elle, à l'augmentation du nombre d'aînés au cours d'une période donnée. Elle relève plutôt d'un flux. Parce qu'ils génèrent des effets territoriaux dissemblables, le vieillissement et la gérontocroissance se doivent d'être différenciés (Dumont, 2016; 2006). Les deux phénomènes appellent à des incidences (et donc à des réponses) différentes en matière d'aménagement et de développement territorial notamment en ce qui a trait aux politiques publiques, lesquelles sont susceptibles d'influer sur les conditions de vie des aînés et sur la perception de leur bien-être, finalité première du développement territorial (Godet et collab. 2018; Morin et Van Den Bussche, 2018; Rican et collab. 2013). Enfin, pouvant être naturelle ou migratoire, la juvénodécroissance désigne la diminution du nombre de jeunes de moins de 24 ans au cours d'une période donnée (Dumont, 2016; 2012).

Le « développement territorial » fait ici référence au processus de changement social pouvant être induit par un ou plusieurs acteurs issus de différents milieux (société civile, État, entreprises,

2. Le vieillissement «par le bas » fait référence à la diminution des effectifs de jeunes à la suite d'une baisse de la natalité qui se traduit par une augmentation de la proportion de personnes âgées, sans nécessairement observer une hausse de l'effectif de ces dernières. 
communautés locales, etc.) qui mettent en place des actions en vue d'améliorer la qualité de vie des individus qui résident au sein de ce territoire suivant une perspective d'équité et de justice sociale (Fortin et Chiasson, 2017; Simard, 2018). Mis en scène sur un territoire, le développement tend à modifier la façon dont les populations tissent leurs relations par rapport à ce dernier, tout en étant influencées par la nature de diverses composantes présentes sur ce même territoire (Simard, 2018). Par conséquent, l'élaboration d'un tel processus suppose la mise en relation de deux types de facteurs : (1) ceux relatifs à la dotation factorielle en ressources, c'est-à-dire le potentiel biophysique, l'organisation de la structure spatiale, l'armature de services, la démographie, l'économie, l'aménagement du territoire, les politiques publiques et l'éducation et (2) ceux inhérents au renforcement des capacités telles que le leadership, la résilience, la concertation des acteurs, l'empowerment, la mobilisation sociale, la gouvernance, le sentiment identitaire, la dynamique relationnelle et communautaire et le capital social (Polèse et collab. 2015; Bisson, 2013; Jean, 2012; Davis, 2008). Cette transversalité est susceptible de créer une synergie unissant les différentes composantes du développement qui se répercute sur le capital socioterritorial, lequel constitue l'aboutissement de ce processus (Capello, 2014).

Qu'il soit permis d'insister sur l'aménagement du territoire comme composante structurelle du processus de développement territorial. Dans le cadre de cet article, la définition de Merlin sera retenue (1988). Selon ce dernier, l'aménagement réfère à des « actions concertées visant à disposer avec ordre les habitants, les activités, les constructions, les équipements et les moyens de communication sur l'étendue d'un territoire » (Merlin, 1988 : 29). Il s'agit, en quelque sorte, de minimiser les nuisances au détriment de la maximisation des complémentarités (Beaudet et Meloche, 2012). Outre la concertation des différents acteurs, l'aménagement implique la mobilisation de ceux-ci dans leurs efforts de planification territoriale, laquelle doit s'effectuer dans le cadre d'une vision prospective (Merlin, 1988). On parlera ainsi d'un aménagement «durable », en référence à l'intégration des composantes environnementale, économique et sociale à cette planification en lien avec le concept de développement durable défini par la Commission Brundtland lors de la formation de la Commission de l'environnement et du développement en 1984 (Commission mondiale sur l'environnement et le développement, 1988). Hormis la protection et la mise en valeur des ressources, l'un des principaux objectifs de l'aménagement consiste à favoriser un équilibre de la structure spatiale de manière à assurer un développement durable, le but étant de promouvoir une certaine forme d'équité entre les individus qui occupent cet espace (Dugas, 1990 ; 1989).

En raison des retombées qu'elle est susceptible de générer, l'économie résidentielle est une dimension qui doit faire partie intégrante de toute politique d'aménagement du territoire. Issue de la théorie de la base économique, l'économie résidentielle « désigne (...) l'offre territoriale visà-vis d'agents résidants inactifs sur le territoire : retraités, touristes, actifs employés ailleurs ( "navetteurs»). La mesure de cette économie s'exprime en valeurs monétaires. Il s'agit donc de montants monétaires de base économique, jusqu'ici largement ignorés des analystes, qui viennent accroître la base productive des territoires (mais aussi les bases publiques, sociales et médicales) » (Davazies, 2009:52). Dès lors, l'économie résidentielle permet de créer des emplois dans le domaine des services, ce qui peut être bénéfique pour plusieurs petites localités rurales du Québec dont l'économie repose sur la mise en valeur des ressources (Segessmann et Crevoisier, 2013; Blasquiet-Revol et collab. 2011; Estèbe et collab. 2007). 


\section{ÉTATS DES LIEUX}

\section{Les personnes âgées}

Loi du nombre oblige, les petites localités figurent parmi le segment de l'espace rural qui fut le moins concerné par la gérontocroissance entre 1986 et 2016 comme en témoigne la figure 1. Au cours de cette période, l'augmentation du nombre de personnes âgées au sein de ces petits milieux $(78,8 \%)$ a été nettement inférieure à la moyenne québécoise (201,5\%). Par ailleurs, le nombre de personnes âgées a plus que quadruplé dans 17 petites localités (cf. tableau 1). Si certaines correspondent à des milieux de villégiature prisés par les aînés en raison de la qualité du cadre de vie et de l'existence d'aménités associées au monde rural, d'autres sont assujetties à une forte dévitalisation reflétant la très grande diversité de l'espace rural (Jean, 2012 ; Simard, 2003). Dans ces milieux, la dénatalité et l'exode ont contribué à gonfler la proportion de personnes âgées. Il s'ensuit que la gérontocroissance affecte les milieux ruraux isolés, sous faible influence urbaine, ou au contraire ceux situés à proximité des pôles urbains qui apparaissent attractifs pour les individus à l'âge de la retraite (Gucher, 2014). Cette dernière situation explique notamment leur taux de migration

FIGURE 1

Croissance, en pourcentage, du nombre de personnes âgées dans les milieux ruraux selon la taille de leur population en 2016, en comparaison avec les milieux urbains et l'ensemble du Québec, 1986 à 2016

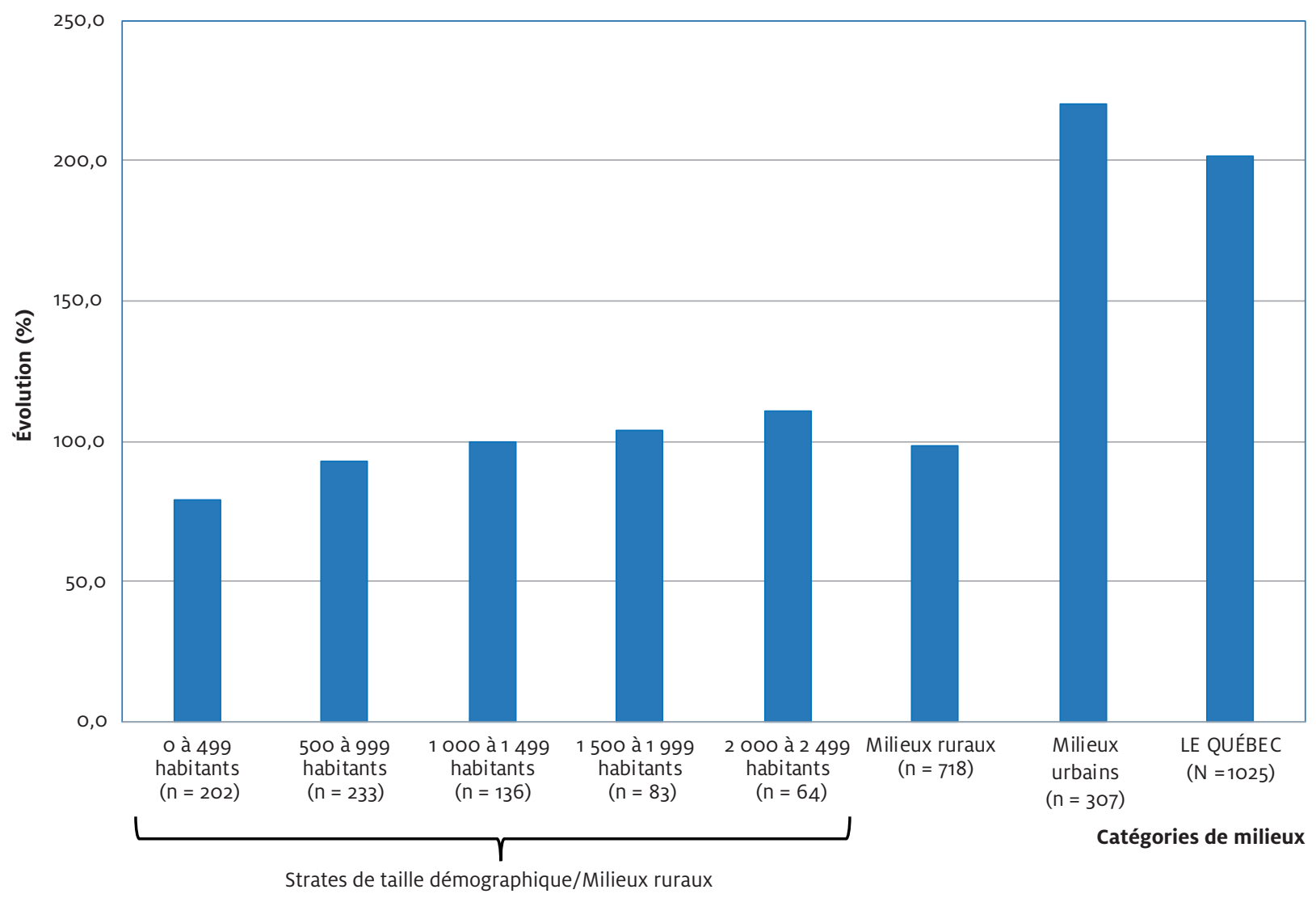


TABLEAU 1

Caractéristiques démographiques et socio-économiques des petites localités rurales du Québec selon le taux de croissance de la population âgée de 65 ans et plus entre 1986 et 2016

\begin{tabular}{|c|c|c|c|c|c|c|c|c|c|}
\hline $\begin{array}{l}\text { Taux de croissance de la } \\
\text { population âgée de } 65 \text { ans } \\
\text { et plus entre } 1986 \text { et } 2016\end{array}$ & $<0 \%$ & ०\% & $\begin{array}{l}\text { de } 0,1 \text { à } \\
99,9 \%\end{array}$ & $\begin{array}{l}\text { de } 100 \text { à } \\
199,9 \%\end{array}$ & $\begin{array}{l}\text { de } 200 \text { à } \\
299,9 \%\end{array}$ & $\begin{array}{l}\text { de } 300 \text { à } \\
399,9 \%\end{array}$ & $\begin{array}{l}>0 u= \\
400 \%\end{array}$ & $\begin{array}{l}\text { Ensemble } \\
\text { des petites } \\
\text { localités }\end{array}$ & \multirow[t]{2}{*}{$\begin{array}{l}\text { Ensemble } \\
\text { des localités } \\
\text { du Québec }\end{array}$} \\
\hline Nombre de localités & 8 & 10 & 85 & 68 & 14 & 9 & 8 & 202 & \\
\hline \multicolumn{10}{|c|}{ Variation des effectifs de population } \\
\hline $\begin{array}{l}\text { Effectif moyen des localités } \\
(2016)\end{array}$ & 351 & 216 & 319 & 350 & 271 & 291 & 271 & 314 & 7937 \\
\hline $\begin{array}{l}\text { Croissance de l'effectif de } \\
\text { la population, 1986-2016 }\end{array}$ & -1096 & -1068 & -8856 & -2990 & 87 & 380 & 499 & -12692 & 1631900 \\
\hline $\begin{array}{l}\text { Croissance, en \%, de la } \\
\text { population, 1986-2016 }\end{array}$ & $-30,9$ & $-35,4$ & $-24,4$ & $-12,3$ & 2,0 & 16,9 & 29,9 & $-16,4$ & 25,0 \\
\hline $\begin{array}{l}\text { Nombre de localités en } \\
\text { décroissance démographique } \\
\text { continuent depuis } 1986\end{array}$ & 1 & 3 & 14 & 8 & o & 2 & o & 28 & 98 \\
\hline $\begin{array}{l}\text { Croissance de l'effectif des } \\
\text { o-24 ans, 1986-2016 }\end{array}$ & -600 & -805 & -8365 & -5245 & -1065 & -395 & -215 & -16690 & 403110 \\
\hline $\begin{array}{l}\text { Croissance, en \%, de l'effectif } \\
\text { des o-24 ans, 1986-2016 }\end{array}$ & $-52,2$ & $-62,2$ & $-56,3$ & $-51,1$ & $-56,1$ & $-41,6$ & $-29,1$ & $-52,9$ & 21,7 \\
\hline $\begin{array}{l}\text { Croissance de l'effectif des } \\
65 \text { ans et plus, 1986-2016 }\end{array}$ & -195 & 0 & 1955 & 2835 & 785 & 500 & 430 & 6310 & 1013620 \\
\hline \multicolumn{10}{|c|}{ Variables socio-économiques } \\
\hline $\begin{array}{l}\text { Taux de migration pendu- } \\
\text { laire (2016) }\end{array}$ & 68,9 & 74,2 & 83,8 & 82,2 & 89,2 & 80,7 & 87,1 & 80,9 & 47,8 \\
\hline Taux d'activité (2016) & 48,7 & 54,1 & 58,3 & 55,8 & 54,6 & 52,6 & 62,4 & 55,2 & 64,1 \\
\hline Taux de chômage (2016) & 9,5 & 16,3 & 9,9 & 12,4 & 9,0 & 11,0 & 8,5 & 10,9 & 7,2 \\
\hline $\begin{array}{l}\% \text { des travailleurs affectés } \\
\text { au secteur primaire (2016) }\end{array}$ & 19,1 & 10,8 & 19,2 & 15,9 & 10,8 & 10,7 & 15,1 & 14,5 & 2,5 \\
\hline $\begin{array}{l}\text { \% des travailleurs affectés } \\
\text { au secteur secondaire } \\
(2016)\end{array}$ & 24,4 & 19,7 & 22,0 & 20,3 & 30,5 & 21,2 & 21,7 & 22,8 & 17,1 \\
\hline $\begin{array}{l}\text { \% des travailleurs affectés } \\
\text { au secteur tertiaire (2016) }\end{array}$ & 56,5 & 69,5 & 58,8 & 63,8 & 58,7 & 68,1 & 63,2 & 62,7 & 80,4 \\
\hline \multicolumn{10}{|c|}{ Variables liées au revenu } \\
\hline $\begin{array}{l}\text { Revenu moyen des familles } \\
\text { en } \$(2016)\end{array}$ & 57924 & $\begin{array}{r}60 \\
245\end{array}$ & 62933 & 65260 & 64651 & 62485 & 69611 & 63301 & 79378 \\
\hline $\begin{array}{l}\text { \% du revenu provenant } \\
\text { d'un emploi (2016) }\end{array}$ & 57,3 & 56,0 & 60,2 & 60,2 & 57,5 & 56,0 & 58,1 & 57,9 & 68,4 \\
\hline $\begin{array}{l}\text { \% du revenu provenant de } \\
\text { transferts gouvernementaux } \\
(2016)\end{array}$ & 30,1 & 31,8 & 26,2 & 24,9 & 25,9 & 26,8 & 20,0 & 26,5 & 15,6 \\
\hline \multicolumn{10}{|c|}{ Variable géographique } \\
\hline $\begin{array}{l}\text { Distance par rapport à la } \\
\text { ville la plus près }(\mathrm{km})\end{array}$ & 31,7 & 34,7 & 30,5 & 32,6 & 29,0 & 21,6 & 23,2 & 29,0 & - \\
\hline
\end{tabular}

Source: Statistique Canada. Recensements de 1986 et de 2016. Compilation et calculs de l'auteur. 
pendulaire très élevé 3 , ce dernier atteignant 87,1 \% au sein des petites localités dont le nombre d'aînés a plus que quadruplé, contre 80,9 \% pour l'ensemble des petites localités.

Huit petites localités ont suivi une trajectoire inverse. Ces milieux en gérontodécroissance se sont caractérisés par un déclin démographique de l'ordre de 30,9\% entre 1986 et 2016. En fait, une seule de ces huit localités, Aupaluk, a connu une croissance de ses effectifs en l'espace de 30 ans. Ce groupe se distingue par la précarité de leur économie comme en témoigne leur faible taux d'activité $(48,1 \%)$, cette dernière étant particulièrement dépendante des activités liées à l'extraction des ressources, le secteur primaire composant 19,8 \% de leur structure occupationnelle. Il se caractérise aussi par la faiblesse de leur niveau de revenu.

L'augmentation de la proportion de personnes âgées de 65 ans et plus a été supérieure à 25 points dans 12 subdivisions de recensement (cf. tableau 2). Ces milieux ont tendance à se concentrer dans les régions périphériques de la province, dont la Gaspésie, qui en compte quatre, ainsi que la Côte-Nord. L'intensité du vieillissement tend à s'amplifier avec la décroissance démographique. Par ailleurs, seulement deux petites localités (Aupaluk et Quaqtaq) ont affiché une évolution négative de leur proportion de personnes âgées entre 1986 et 2016, et ce, bien qu'elles aient gagné cinq aînés au cours de cette période.

\section{Les jeunes}

Les petites localités sont aussi particulièrement affectées par la juvénodécroissance. Entre 1986 et 2016, elles ont perdu 16690 jeunes, soit une baisse de 52,9\%. Sur les 202 petites localités à l'étude, 31 ont perdu plus des trois quarts de leurs jeunes en 30 ans. De ce nombre, 17 se situent dans la région de l'Est-du-Québec (cf. tableau 3). Ces milieux souffrent d'une économie anémique comme en témoigne leur faible taux d'activité $(46,8 \%)$. Ils se caractérisent aussi par la faiblesse de leur niveau de revenu (57 825 \$) comparativement à celui des autres catégories. Seulement 12 petites localités, dont sept correspondent à des réserves autochtones, ont accusé une augmentation du nombre de jeunes entre 1986 et 2016. Le pourcentage d'augmentation oscille entre 14,3\% à Kitcisakik à $400 \%$ dans le cas d'Estérel. Mentionnons que le nombre de jeunes est demeuré inchangé au sein d'une seule petite localité, à savoir celle de Rapide-Danseur dans la municipalité régionale de comté (MRC) Abitibi-Ouest.

Abritant une moyenne de 314 habitants, les 12 petites localités en juvénocroissance se sont distinguées par une très forte augmentation $(108,6 \%)$ de leurs effectifs démographiques entre 1986 et 2016. De 725 qu'ils étaient en 1986, le nombre de jeunes est passé à 1215 en 2016, ce qui représente une augmentation de 67,6 \%. Par ailleurs, la proportion de jeunes a décru de 8,6 points au cours de la période à l'étude. Seule la localité d'Estérel fait bande à part, cette même proportion ayant augmenté de 3,8 points. Hormis Quaqtaq et Aupaluk, ces milieux en juvénocroissance sont également touchés par le vieillissement «par le haut», la part des 65 ans et plus ayant passé de

3. Le taux de migration pendulaire correspond au rapport entre la population active de 15 ans et plus occupant un emploi à l'extérieur de la subdivision de recensement et la population active totale de cette même subdivision, le tout multiplié par 100. 
TABLEAU 2

Caractéristiques démographiques et socio-économiques des petites localités rurales du Québec selon le taux de croissance de la proportion de personnes âgées de 65 ans et plus entre 1986 et 2016

\begin{tabular}{|c|c|c|c|c|c|c|c|c|c|}
\hline $\begin{array}{l}\text { Taux de croissance de la } \\
\text { proportion de personnes } \\
\text { âgées de } 65 \text { ans et plus } \\
\text { entre } 1986 \text { et } 2016\end{array}$ & $>25 \%$ & $\begin{array}{l}20,1 \text { à } \\
25 \%\end{array}$ & $\begin{array}{l}15,1 \text { à } \\
20 \%\end{array}$ & $\begin{array}{c}10,1 \text { à } \\
15 \%\end{array}$ & $\begin{array}{l}5,1 \text { à } \\
10 \%\end{array}$ & $\begin{array}{c}0,1 \text { à } \\
5 \%\end{array}$ & $<0$ & $\begin{array}{l}\text { Ensemble } \\
\text { des petites } \\
\text { localités }\end{array}$ & $\begin{array}{c}\text { Ensemble } \\
\text { des localités } \\
\text { du Québec }\end{array}$ \\
\hline Nombre de localités & 12 & 22 & 34 & 58 & 54 & 20 & 2 & 202 & \\
\hline \multicolumn{10}{|c|}{ Variation des effectifs de population } \\
\hline $\begin{array}{l}\text { Effectif moyen des localités } \\
(2016)\end{array}$ & 216 & 246 & 312 & 336 & 337 & 317 & 306 & 314 & 7937 \\
\hline $\begin{array}{l}\text { Croissance de l'effectif de la } \\
\text { population, 1986-2016 }\end{array}$ & -1204 & -1375 & -3566 & -4402 & -2215 & -247 & 317 & -12692 & 1631900 \\
\hline $\begin{array}{l}\text { Croissance, en } \% \text {, de la } \\
\text { population, 1986-2016 }\end{array}$ & $-31,8$ & $-21,0$ & $-25,2$ & $-18,2$ & $-10,8$ & $-3,7$ & 107,5 & $-16,4$ & 25,0 \\
\hline $\begin{array}{l}\text { Nombre de localités en } \\
\text { décroissance démographique } \\
\text { continue depuis } 1986\end{array}$ & 4 & 3 & 4 & 10 & 3 & 3 & o & 28 & 98 \\
\hline $\begin{array}{l}\text { Croissance de l'effectif des } \\
\text { o-24 ans, 1986-2016 }\end{array}$ & -1035 & -1835 & -3980 & -5515 & -3550 & -835 & 150 & -16690 & 403110 \\
\hline $\begin{array}{l}\text { Croissance, en \%, de l'effectif } \\
\text { des o-24 ans, 1986-2016 }\end{array}$ & $-78,1$ & $-65,9$ & -67.3 & $-54,4$ & -42.8 & $-30,7$ & 78,9 & $-52,9$ & 21,7 \\
\hline $\begin{array}{l}\text { Croissance de l'effectif des } \\
65 \text { ans et plus, 1986-2016 }\end{array}$ & 595 & 1025 & 1500 & 2130 & 1000 & 140 & 5 & 6310 & 1013620 \\
\hline \multicolumn{10}{|c|}{ Variables socio-économiques } \\
\hline $\begin{array}{l}\text { Taux de migration pendulaire } \\
(2016)\end{array}$ & 66,5 & 88,2 & 82,1 & 84,5 & 84,0 & 78,9 & 19,8 & 80,9 & 47,8 \\
\hline Taux d'activité (2016) & 42,2 & 48,8 & 52,2 & 56,6 & 62,0 & 59,2 & 79,1 & 55,2 & 64,1 \\
\hline Taux de chômage (2016) & 16,6 & 13,7 & 12,9 & 11,9 & 7,2 & 9,5 & 20,3 & 10,9 & 7,2 \\
\hline $\begin{array}{l}\text { \% des travailleurs affectés au } \\
\text { secteur primaire (2016) }\end{array}$ & 8,0 & 13,9 & 15,1 & 17,7 & 19,8 & 13,4 & 3,2 & 14,5 & 2,5 \\
\hline $\begin{array}{l}\text { \% des travailleurs affectés au } \\
\text { secteur secondaire (2016) }\end{array}$ & 24,0 & 24,1 & 27,1 & 19,2 & 21,8 & 24,0 & 0,0 & 22,8 & 17,1 \\
\hline $\begin{array}{l}\text { \% des travailleurs affectés au } \\
\text { secteur tertiaire (2016) }\end{array}$ & 68,0 & 62,0 & 57,8 & 63,1 & 58,4 & 66,2 & 96,8 & 62,7 & 80,4 \\
\hline \multicolumn{10}{|c|}{ Variables liées au revenu } \\
\hline $\begin{array}{l}\text { Revenu moyen des familles } \\
\text { économiques en } \$(2016)\end{array}$ & 54640 & 63003 & 58149 & 63553 & 68570 & 63618 & 75648 & 63301 & 79378 \\
\hline $\begin{array}{l}\text { \% du revenu provenant d'un } \\
\text { emploi (2016) }\end{array}$ & 41,7 & 52,8 & 54,4 & 59,5 & 64,7 & 61,5 & 79,1 & 57,9 & 68,4 \\
\hline $\begin{array}{l}\text { \% du revenu provenant de } \\
\text { transferts gouvernementaux } \\
(2016)\end{array}$ & 35,8 & 24,4 & 30,7 & 26,3 & 22,3 & 25,0 & 16,7 & 26,5 & 15,6 \\
\hline \multicolumn{10}{|c|}{ Variable géographique } \\
\hline $\begin{array}{l}\text { Distance par rapport à la ville } \\
\text { la plus près }(\mathrm{km})\end{array}$ & 45,9 & 32,9 & 37,1 & 29,6 & 27,8 & 27,8 & 37,9 & 29,0 & - \\
\hline
\end{tabular}

Source: Statistique Canada. Recensements de 1986 et de 2016. Compilation et calculs de l'auteur. 
TABLEAU 3

Caractéristiques démographiques et socio-économiques des petites localités rurales du Québec selon le taux de croissance de la population âgée de 0-24 ans entre 1986 et 2016

\begin{tabular}{|c|c|c|c|c|c|c|c|}
\hline $\begin{array}{l}\text { Taux de croissance de la population } \\
\text { âgée de } 0-24 \text { ans entre } 1986 \text { et } 2016\end{array}$ & $<-75 \%$ & $\begin{array}{c}-50 \\
\text { à } 74,9 \%\end{array}$ & $\begin{array}{l}-25 \\
\text { à } 49,0 \%\end{array}$ & $\begin{array}{c}-0,1 \text { à } \\
24,9 \%\end{array}$ & $>0 \%$ & $\begin{array}{c}\text { Ensemble } \\
\text { des petites } \\
\text { localités }\end{array}$ & \multirow[t]{2}{*}{$\begin{array}{c}\text { Ensemble } \\
\text { des localités } \\
\text { du Québec }\end{array}$} \\
\hline Nombre de localités & 31 & 83 & 64 & 11 & 12 & $201^{\mathrm{a}}$ & \\
\hline \multicolumn{8}{|c|}{ Variation des effectifs de population } \\
\hline Effectif moyen des localités (2016) & 210 & 318 & 360 & 367 & 314 & 314 & 7937 \\
\hline $\begin{array}{l}\text { Croissance de l'effectif de la population, } \\
1986-2016\end{array}$ & -5343 & -8375 & -1801 & 818 & 1801 & -12692 & 1631900 \\
\hline $\begin{array}{l}\text { Croissance, en \%, de la population, } \\
1986-2016\end{array}$ & $-45,1$ & $-24,6$ & $-7,4$ & 25,5 & 108,6 & $-16,4$ & 25,0 \\
\hline $\begin{array}{l}\text { Nombre de localités en décroissance } \\
\text { démographique continue depuis } 1986\end{array}$ & 12 & 14 & 4 & O & $\mathrm{O}$ & 28 & 98 \\
\hline $\begin{array}{l}\text { Croissance de l'effectif des 0-24 ans, } \\
1986-2016\end{array}$ & -4040 & -8690 & -4180 & -240 & 490 & -16690 & 403110 \\
\hline $\begin{array}{l}\text { Croissance, en \%, de l'effectif des 0-24 } \\
\text { ans, 1986-2016 }\end{array}$ & $-80,2$ & $-61,3$ & $-41,5$ & $-18,8$ & 67,6 & $-52,9$ & 21,7 \\
\hline $\begin{array}{l}\text { Croissance de l'effectif des } 65 \text { ans et } \\
\text { plus, } 1986-2016\end{array}$ & 855 & 2625 & 2130 & 430 & 380 & -6310 & 1013620 \\
\hline \multicolumn{8}{|c|}{ Variables socio-économiques } \\
\hline Taux de migration pendulaire (2016) & 74,6 & 81,7 & 88,9 & 84,7 & 60,2 & 80,9 & 47,8 \\
\hline Taux d'activité (2016) & 46,8 & 55,5 & 60,0 & 58,4 & 64,3 & 55,2 & 64,1 \\
\hline Taux de chômage (2016) & 19,2 & 11,6 & 8,6 & 5,5 & 12,3 & 10,9 & 7,2 \\
\hline $\begin{array}{l}\% \text { des travailleurs affectés au secteur } \\
\text { primaire (2016) }\end{array}$ & 15,9 & 17,5 & 18,8 & 10,9 & 4,5 & 14,5 & 2,5 \\
\hline $\begin{array}{l}\text { \% des travailleurs affectés au secteur } \\
\text { secondaire (2016) }\end{array}$ & 22,1 & 24,0 & 20,9 & 25,2 & 11,6 & 22,8 & 17,1 \\
\hline $\begin{array}{l}\text { \% des travailleurs affectés au secteur } \\
\text { tertiaire (2016) }\end{array}$ & 62,0 & 58,5 & 60,3 & 63,9 & 83,9 & 62,7 & 80,4 \\
\hline \multicolumn{8}{|c|}{ Variables liées au revenu } \\
\hline Revenu moyen des familles en \$ (2016) & 57825 & 61387 & 65885 & 66449 & 72960 & 63301 & 79378 \\
\hline $\begin{array}{l}\text { \% du revenu provenant d'un emploi } \\
\text { (2016) }\end{array}$ & 48,7 & 58,3 & 61,8 & 60,9 & 64,6 & 57,9 & 68,4 \\
\hline $\begin{array}{l}\text { \% du revenu provenant de transferts } \\
\text { gouvernementaux (2016) }\end{array}$ & 36,6 & 28,1 & 23,2 & 22,5 & 20,2 & 26,5 & 15,6 \\
\hline \multicolumn{8}{|c|}{ Variable géographique } \\
\hline $\begin{array}{l}\text { Distance par rapport à la ville la plus } \\
\text { près }(\mathrm{km})\end{array}$ & 43,9 & 28,7 & 26,5 & 32,7 & 24,7 & 29,0 & - \\
\hline
\end{tabular}

a Le total est de 201 petites localités (au lieu de 202), car nous avons retiré Notre-Dame-des-Anges, une municipalité gérée par les Augustines de la Miséricorde de Jésus de l'Hôpital général de Québec qui ne comportait aucun jeune, et ce, tant en 1986 qu'en 2016.

Source: Statistique Canada. Recensements de 1986 et de 2016. Compilation et calculs de l'auteur. 
$8,7 \%$ en 1986 à 15,2 \% en 2016. Sur le plan économique, ces milieux se distinguent par la forte dominance du secteur tertiaire qui accapare 83,9\% des actifs. Les activités liées aux services semblent contribuer au dynamisme de l'économie puisque ce groupe est celui qui, toutes catégories de petites localités confondues, affiche le taux d'activité $(64,3 \%)$ le plus élevé. Bon nombre des emplois disponibles au sein de ces milieux sont occupés à l'intérieur de la localité de séjour, le taux de migration pendulaire ne s'établissant qu'à 60,2 \%. Précisons que près des deux tiers du revenu de ces petites localités proviennent d'un emploi. Couplée au fait que les transferts gouvernementaux ne composent que $20,2 \%$ du revenu total, cette situation témoigne du dynamisme économique qui prévaut au sein de ces milieux.

À l'autre bout du spectre, les petites localités en très forte juvénodécroissance présentent un profil socio-économique différent de leurs homologues du groupe précédent. Quelque 31 milieux de petite taille démographique se sont caractérisés par une diminution de $75 \%$ ou plus de la population âgée de o à 24 ans entre 1986 et 2016. Parmi ces 31 entités, 17 se situent dans la région de l'Est-du-Québec, dont 10 au Bas-Saint-Laurent. Les autres se dispersent sur la CôteNord, en Abitibi-Témiscamingue, dans la région Chaudière-Appalaches et en Haute-Mauricie. Ces petites localités abritent en moyenne 210 personnes. En l'espace de 30 ans, elles ont perdu presque la moitié de leurs effectifs démographiques. La très forte diminution du nombre de jeunes a eu pour effet d'intensifier le vieillissement tant «par le bas » que " par le haut». Sur le plan économique, leur taux d'activité moyen s'établit à 46,8 \% en 2016. Quant à la composition de la structure occupationnelle, elle est dominée par le secteur tertiaire qui accapare $62 \%$ des actifs. Ainsi, ces milieux se caractérisent par leur fragilité, celle-ci se manifestant notamment par la faiblesse du revenu moyen des familles. Les transferts gouvernementaux composent 36,6\% du revenu total, ce qui constitue un autre signe de leur vulnérabilité économique. Finalement, soulignons que plusieurs de ces petites localités souffrent d'enclavement géographique en plus d'être aux prises avec une très forte dispersion de leur population. En effet, 11 de ces petits milieux se retrouvent à plus de 50 kilomètres d'une ville. Leur distance moyenne par rapport à celle-ci s'établit à 43,9 kilomètres.

Ce qui apparaît encore plus inquiétant est l'évolution de la proportion de jeunes au sein des petites localités rurales du Québec. De 41,2 \% qu'elle était en 1986, celle-ci est passée à 23,3 \% en 2016, soit une baisse de 17,9 points. On dénombrait 10 petites localités dont la proportion de jeunes a décru de 30 points ou plus entre 1986 et 2016 ( cf. tableau 4). Elles ont accusé une perte de 1096 habitants entre 1986 et 2016, soit une diminution de 37,1\%. De son côté, la part des personnes âgées de 65 ans et plus parmi la population totale s'est accrue de 18,5 points en l'espace de 30 ans. Il s'agit d'un gain de 365 individus par rapport à 1986. Le sous-emploi et, de façon générale, la précarité de l'économie auxquels ces milieux sont assujettis ne sont sûrement pas de nature à attirer ou retenir les jeunes.

Seulement quatre petites localités ont accru leur proportion de jeunes entre 1986 et 2016 alors qu'une seule n'a subi aucun changement. Alors que ces quatre municipalités ne comportaient aucun jeune en 1986, elles s'en sont enrichies de 150 en 2016. À l'exception d'Estérel, ces petites localités, qui abritent en moyenne 122 habitants, sont isolées de la trame de peuplement, celles-ci se situant en moyenne à 51,9 kilomètres d'une ville. En 2016, les aînés représentaient 30,7 \% de la 
TABLEAU 4

Caractéristiques démographiques et socio-économiques des petites localités rurales du Québec selon le taux de croissance de la proportion de personnes âgées de o-24 ans entre 1986 et 2016

\begin{tabular}{|c|c|c|c|c|c|c|c|}
\hline $\begin{array}{l}\text { Taux de croissance de la proportion de } \\
\text { personnes âgées de 0-24 ans entre } 1986 \\
\text { et } 2016\end{array}$ & $>0 \%$ & $\begin{array}{l}-0,1 \text { à } \\
-9,9 \%\end{array}$ & $\begin{array}{c}-10 \text { à } \\
-19,9 \%\end{array}$ & $\begin{array}{l}-20 \text { à } \\
-29,9 \%\end{array}$ & $-30<$ & $\begin{array}{l}\text { Ensemble } \\
\text { des petites } \\
\text { localités }\end{array}$ & \multirow[t]{2}{*}{$\begin{array}{l}\text { Ensemble } \\
\text { des localités } \\
\text { du Québec }\end{array}$} \\
\hline Nombre de localités & 4 & 14 & 101 & 72 & 10 & $201^{\mathrm{a}}$ & \\
\hline \multicolumn{8}{|c|}{ Variation des effectifs de population } \\
\hline Effectif moyen des localités (2016) & 122 & 351 & 338 & 301 & 186 & 314 & 7937 \\
\hline $\begin{array}{l}\text { Croissance de l'effectif de la population, 1986- } \\
2016\end{array}$ & 382 & 681 & -5199 & -7306 & -1096 & -12692 & 1631900 \\
\hline Croissance, en \%, de la population, 1986-2016 & 360,4 & 16,1 & $-13,2$ & $-25,2$ & $-37,1$ & $-16,4$ & 25,0 \\
\hline $\begin{array}{l}\text { Nombre de localités en décroissance } \\
\text { démographique continue depuis } 1986\end{array}$ & o & 2 & 12 & 14 & o & 28 & 98 \\
\hline $\begin{array}{l}\text { Croissance de l'effectif des 0-24 ans, 1986- } \\
2016\end{array}$ & 80 & -25 & -7365 & -8160 & -1130 & -16690 & 403110 \\
\hline $\begin{array}{l}\text { Croissance, en \%, de l'effectif des 0-24 ans, } \\
\text { 1986-2016 }\end{array}$ & 1600,0 & $-1,5$ & $-46,3$ & $-66,1$ & $-77,7$ & $-52,9$ & 21,7 \\
\hline $\begin{array}{l}\text { Croissance de l'effectif des } 65 \text { ans et plus, } \\
1986-2016\end{array}$ & 140 & 280 & 2930 & 2900 & 265 & -6310 & 1013620 \\
\hline \multicolumn{8}{|c|}{ Variables socio-économiques } \\
\hline Taux de migration pendulaire (2016) & 92,7 & 74,9 & 86,2 & 79,9 & 71,6 & 80,9 & 47,8 \\
\hline Taux d'activité (2016) & 50,3 & 63,8 & 58,6 & 53,1 & 55,1 & 55,2 & 64,1 \\
\hline Taux de chômage (2016) & o & 9,6 & 8,8 & 14,6 & 11,0 & 10,9 & 7,2 \\
\hline $\begin{array}{l}\text { \% des travailleurs affectés au secteur primaire } \\
(2016)\end{array}$ & 7,2 & 11,6 & 17,5 & 17,3 & 10,9 & 14,5 & 2,5 \\
\hline $\begin{array}{l}\text { \% des travailleurs affectés au secteur } \\
\text { secondaire (2016) }\end{array}$ & 12,0 & 15,5 & 21,2 & 24,2 & 29,2 & 22,8 & 17,1 \\
\hline $\begin{array}{l}\text { \% des travailleurs affectés au secteur tertiaire } \\
(2016)\end{array}$ & 61,5 & 72,8 & 61,3 & 58,6 & 59,9 & 62,7 & 80,4 \\
\hline \multicolumn{8}{|c|}{ Variables liées au revenu } \\
\hline Revenu moyen des familles en $\$$ (2016) & n.d & 70388 & 64002 & 62224 & 67606 & 63301 & 79378 \\
\hline \% du revenu provenant d'un emploi (2016) & n.d & 65,5 & 60,4 & 56,9 & 23,5 & 57,9 & 68,4 \\
\hline $\begin{array}{l}\text { \% du revenu provenant de transferts } \\
\text { gouvernementaux (2016) }\end{array}$ & n.d & 21,2 & 24,8 & 28,7 & 9,4 & 26,5 & 15,6 \\
\hline \multicolumn{8}{|c|}{ Variable géographique } \\
\hline Distance par rapport à la ville la plus près $(\mathrm{km})$ & 51,9 & 30,0 & 26,4 & 34,6 & 42,8 & 29,0 & \\
\hline
\end{tabular}

${ }^{a}$ Le total est de 201 petites localités (au lieu de 202), car nous avons retiré Notre-Dame-des-Anges, une municipalité gérée par les Augustines de la Miséricorde de Jésus de l'Hôpital général de Québec qui ne comportait aucun jeune, et ce, tant en 1986 qu'en 2016.

n.d. donnée non disponible

Source: Statistique Canada. Recensements de 1986 et de 2016. Compilation et calculs de l'auteur. 
population comparativement à 9,4\% en 1986. Leur économie semble dépendre de celle de leur environnement immédiat, car le taux de migration pendulaire, en dépit de leur éloignement par rapport à la ville, s'établit à 92,7\%.

\section{MÉTHODE}

Pour chacune des petites localités rurales du Québec, un indice synthétique a été construit afin d'illustrer l'état de leur situation en considérant la dynamique évolutive de la gérontocroissance, du vieillissement "par le haut ", du vieillissement " par le bas ", ainsi qu'eu égard à l'intensité de la décroissance démographique au cours de la période 1986-2016. Certes, force est d'admettre que la méthode des indices est sujette à des critiques notamment en ce qui a trait à la subjectivité concernant le choix et la pondération des composantes, si bien que cette approche «tend à remettre en cause la légitimité locale de ces indicateurs comme outils d'orientation des acteurs publics et de leur suivi » (Dialga et Le, 2014:4). Néanmoins, en plus d'être utilisée par plusieurs géographes (Simard, 2015a ; Houée, 1996; Bontron et Cabanis 1993; Gumuchian, 1990), cette méthode permet d'éclairer les décideurs dans l'élaboration de politiques publiques et d'évaluer leurs retombées à divers échelons territoriaux en plus de servir d'outil de comparaison.

Bien que cette approche comporte une certaine part d'arbitraire en ce qui concerne la détermination des seuils de classes, les indices ont été pondérés en fonction de leur importance eu égard aux différents phénomènes étudiés comme l'illustre le tableau $5^{4}$.

Dès lors, un indice de 1 est attribué aux petites localités dont la proportion d'aînés s'est accrue de 25 points ou plus entre 1986 et 2016 ainsi qu'à celles dont le nombre d'aînés a plus que quadruplé. Quant à la décroissance démographique, elle a été mesurée en fonction de son intensité. Ainsi, un indice de 4 est attribué aux petites localités dont la décroissance démographique a été supérieure à $45 \%$. Celles dont la diminution a oscillé entre - 30 et - 44,9 \% se sont vues attribuer l'indice 3,2. L’indice 2,5 est par ailleurs attribué aux entités dont la décroissance a varié entre - 15 et - 29,9\% alors que celles dont la diminution a oscillé entre - 0,1 et - 14,9\% ont reçu l'indice 1,6. Toutes les petites localités qui ne figuraient pas dans l'une ou l'autre de ces catégories se sont vues accorder l'indice o. L'indice synthétique correspond à la somme des différents indices partiels pour chacune des entités géographiques à l'étude.

Pour ce qui est des limites de classes, elles ont été déterminées, pour chacune des dynamiques évolutives, en cherchant à établir des regroupements homogènes en fonction de leurs caractéristiques dominantes. L'étendue des classes a été calculée de la manière suivante : xmax - xmin/

4. Bien que les pondérations attribuées aux différentes réalités démographiques comportent une certaine part de subjectivité, certains motifs ont tout de même guidé le choix de cette pondération. Par exemple, force est de reconnaître que le dépeuplement, particulièrement au sein des petites localités, est susceptible d'entraîner davantage de conséquences négatives (notamment en matière de maintien et de la livraison des services de proximité) que le vieillissement "par le haut », ce dernier phénomène pouvant même favoriser, à certains endroits, une diversification de l'économie (essor de l'économie résidentielle, élargissement de l'offre de loisirs, etc.). 
TABLEAU 5

Indice partiel attribué aux petites localités rurales du Québec en fonction de la dynamique évolutive de leur situation démographique

\begin{tabular}{|c|c|c|}
\hline Situation démographique & Dynamique évolutive & Indice partiel \\
\hline $\begin{array}{l}\text { Forte croissance de la proportion de } \\
\text { personnes âgées de } 65 \text { ans et plus }\end{array}$ & $\begin{array}{l}\text { Localité dont la proportion de personnes âgées de } \\
65 \text { ans et plus s'est accrue de } 25 \text { points de pourcentage } \\
\text { ou plus entre } 1986 \text { et } 2016\end{array}$ & 1 \\
\hline Gérontocroissance & Localité dont le nombre d’aînés a plus que quadruplé & 1 \\
\hline $\begin{array}{l}\text { Forte décroissance de la proportion } \\
\text { des } 0-24 \text { ans }\end{array}$ & $\begin{array}{l}\text { Localité dont la proportion de jeunes a diminué de } \\
25 \text { points de pourcentage ou plus entre } 1986 \text { et } 2016\end{array}$ & 1,5 \\
\hline \multirow[t]{4}{*}{ Décroissance démographique } & $\begin{array}{l}\text { Localité dont la population a diminué de } 45 \% \text { ou plus } \\
\text { de } 1986 \text { à } 2016\end{array}$ & 4 \\
\hline & $\begin{array}{l}\text { Localité dont la population a diminué entre } 30 \text { et } \\
44,9 \% \text { de } 1986 \text { à } 2016\end{array}$ & 3,2 \\
\hline & $\begin{array}{l}\text { Localité dont la population a diminué entre } 15 \text { et } \\
29,9 \% \text { de } 1986 \text { à } 2016\end{array}$ & 2,5 \\
\hline & $\begin{array}{l}\text { Localité dont la population a diminué entre o,1 et } \\
14,9 \% \text { de } 1986 \text { à } 2016\end{array}$ & 1,6 \\
\hline
\end{tabular}

nombre de classes. L'écart par rapport à la moyenne pour chacune des dynamiques évolutives a aussi été pris en compte. Il s'en est suivi une classification en quatre types :

- les petites localités dont la situation démographique est extrêmement préoccupante (indice de 7,6 et plus);

- les petites localités dont la situation démographique est très précaire (indice oscillant entre $5,1$ et 7,5$)$;

- les petites localités dont la situation démographique est précaire (indice oscillant entre 2,6 et $5,0)$;

- les petites localités dont la situation démographique est plus ou moins précaire (indice oscillant entre 0,1 et 2,5).

Effectuée sous l'angle géographique, l'analyse mettra l'accent sur les spécificités de chaque typologie en considérant certaines caractéristiques de la structure physico-spatiale ainsi qu'en fonction d'indicateurs liés à l'économie et au niveau de revenu.

\section{ANALYSE DES RÉSULTATS}

Suivant l'élaboration de l'indice de précarité démographique, sur les 202 petites localités rurales du Québec, 81 peuvent être considérées comme étant problématiques. Parmi celles-ci, 13 présentent un profil démographique extrêmement préoccupant alors que huit sont en situation de très grande précarité. Ces 21 entités géographiques sont identifiées par leur toponyme à la figure 2 . On en retrouve cinq au Bas-Saint-Laurent et en Gaspésie ainsi que deux en Abitibi-Témiscamingue et 
FIGURE 2

Précarité démographique des petites localités rurales en fonction de l'évolution de certains paramètres liés au vieillissement et à la décroissance démographique entre 1986 et 2016

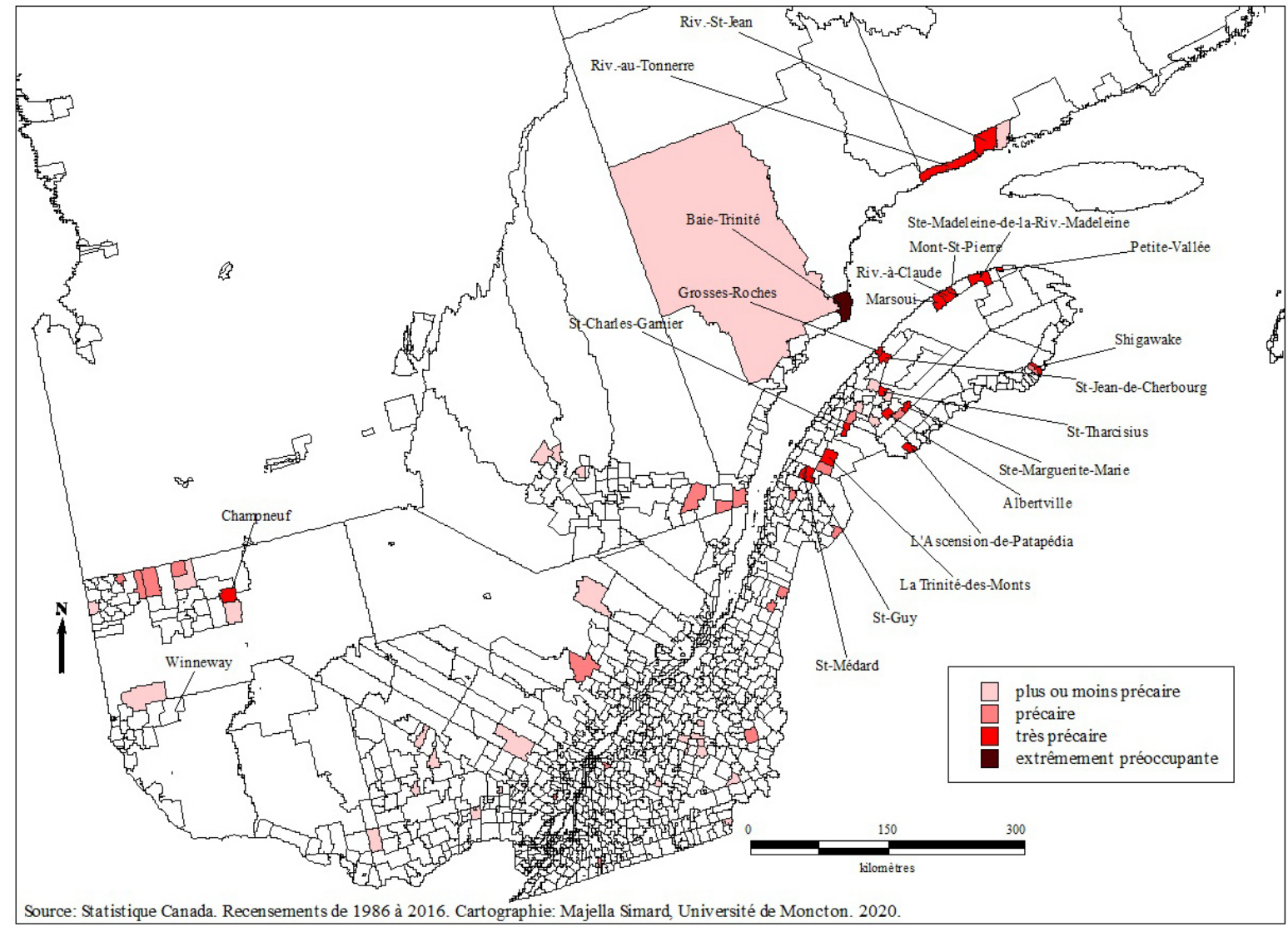

sur la Haute-Côte-Nord (figure 2). Dans ces milieux, la proportion d'aînés s'est accrue de 22,4 points entre 1986 et 2016 (cf. tableau 6). Par ailleurs, ces entités sont beaucoup moins affectées par la gérontocroissance. En 30 ans, elles ne se sont enrichies que de 330 personnes âgées, ce qui représente une augmentation de 64,1\%. Ces 13 petites localités ont une population moyenne de 202 habitants. Par ailleurs, au cours de la période à l'étude, elles ont perdu la moitié de leurs effectifs. Huit sont en décroissance démographique continue depuis 1986. En l'espace de 30 ans, elles ont perdu 1775 jeunes. En valeur relative, la proportion des o à 24 ans a décru de 26,9 points. Ces milieux se distinguent aussi par la précarité de leur économie, dont le taux de chômage moyen s'établissait à $27 \%$ en 2016. La faiblesse de l'activité économique se traduit par une surreprésentation du secteur secondaire dans la composition de leur structure occupationnelle. En effet, celui-ci retient 29,1\% des actifs en comparaison à $23 \%$ pour l'ensemble des petites localités du Québec. De même, les services ne retiennent que 54,2 \% des actifs. Dès lors, l'économie de ces localités est fortement dépendante des transferts gouvernementaux, lesquels composent $40,3 \%$ du revenu total. Cette situation a pour corollaire la faible proportion du revenu provenant d'un emploi 
TABLEAU 6

Caractéristiques démographiques et socio-économiques des petites localités rurales du Québec selon leur niveau de précarité démographique

\begin{tabular}{|c|c|c|c|c|c|c|}
\hline $\begin{array}{l}\text { Niveau de précarité } \\
\text { démographique }\end{array}$ & $\begin{array}{c}\text { Extrêmement } \\
\text { préoccupant } \\
(n=13)\end{array}$ & $\begin{array}{l}\text { Très précaire } \\
\qquad(\mathrm{n}=8)\end{array}$ & $\begin{array}{l}\text { Précaire } \\
(n=20)\end{array}$ & $\begin{array}{l}\text { Plus ou moins } \\
\text { précaire } \\
(n=40)\end{array}$ & $\begin{array}{c}\text { Ensemble } \\
\text { des petites } \\
\text { localités }\end{array}$ & $\begin{array}{l}\text { Ensemble des } \\
\text { localités du } \\
\text { Québec }\end{array}$ \\
\hline \multicolumn{7}{|c|}{ Variation des effectifs de la population } \\
\hline Effectif moyen des localités (2016) & 202 & 226 & 278 & 306 & 314 & 7937 \\
\hline $\begin{array}{l}\text { Croissance de l'effectif de la popula- } \\
\text { tion, } 1986-2016\end{array}$ & -2623 & -1711 & -2785 & -323 & -12692 & 1631900 \\
\hline $\begin{array}{l}\text { Croissance, en \%, de la population, } \\
\text { 1986-2016 }\end{array}$ & $-50,0$ & $-48,6$ & $-33,3$ & $-2,6$ & $-16,4$ & 25,0 \\
\hline $\begin{array}{l}\text { Nombre de localités en décroissance } \\
\text { démographique continue depuis } 1986\end{array}$ & 8 & 4 & 8 & 7 & 28 & 98 \\
\hline $\begin{array}{l}\text { Croissance de l'effectif des o-24 ans, } \\
1986-2016\end{array}$ & -1775 & -1115 & -2445 & -2735 & -16690 & 403110 \\
\hline $\begin{array}{l}\text { Croissance, en \%, de l'effectif des } \\
\text { o-24 ans, 1986-2016 }\end{array}$ & $-82,9$ & $-75,6$ & $-67,8$ & $-51,2$ & $-52,9$ & 21,7 \\
\hline $\begin{array}{l}\text { Croissance de l'effectif des } 65 \text { ans et } \\
\text { plus, 1986-2016 }\end{array}$ & 330 & 125 & 630 & 1890 & 6310 & 1013620 \\
\hline $\begin{array}{l}\text { Croissance, en \%, de l'effectif des } \\
65 \text { ans et plus, 1986-2016 }\end{array}$ & 64,1 & 32,9 & 83,4 & 176,6 & 187,1 & 210,5 \\
\hline \multicolumn{7}{|c|}{ Variables socio-économiques } \\
\hline Taux de migration pendulaire (2016) & 67,0 & 78,8 & 83,8 & 79,4 & 80,9 & 47,8 \\
\hline Taux d'activité (2016) & 42,2 & 43,1 & 54,9 & 63,1 & 55,2 & 64,1 \\
\hline Taux de chômage (2016) & 27,0 & 19,2 & 9,7 & 10,0 & 10,9 & 7,2 \\
\hline $\begin{array}{l}\% \text { des travailleurs affectés au secteur } \\
\text { primaire (2016) }\end{array}$ & 16,7 & 18,9 & 17,6 & 17,8 & 14,5 & 2,5 \\
\hline $\begin{array}{l}\% \text { des travailleurs affectés au secteur } \\
\text { secondaire (2016) }\end{array}$ & 29,1 & 17,7 & 22,3 & 18,1 & 22,8 & 17,1 \\
\hline $\begin{array}{l}\text { \% des travailleurs affectés au secteur } \\
\text { tertiaire (2016) }\end{array}$ & 54,2 & 63,4 & 60,1 & 64,1 & 62,7 & 80,4 \\
\hline \multicolumn{7}{|c|}{ Variables liées au revenu } \\
\hline $\begin{array}{l}\text { Revenu moyen des familles } \\
\text { économiques en } \$(2016)\end{array}$ & 51584 & 61184 & 59245 & 64548 & 63301 & 79378 \\
\hline $\begin{array}{l}\text { \% du revenu provenant d'un emploi } \\
\text { (2016) }\end{array}$ & 42,4 & 51,7 & 56,9 & 58,8 & 57,9 & 68,5 \\
\hline $\begin{array}{l}\text { \% du revenu provenant de transferts } \\
\text { gouvernementaux (2016) }\end{array}$ & 40,3 & 36,6 & 30,8 & 25,6 & 26,5 & 15,6 \\
\hline \multicolumn{7}{|c|}{ Autres } \\
\hline $\begin{array}{l}\text { Distance par rapport à la ville la plus } \\
\text { près (en } \mathrm{km} \text { ) }\end{array}$ & 40,5 & 44,5 & 35,4 & 32,2 & 29,0 & - \\
\hline $\begin{array}{l}\text { Nombre de localités faisant partie du } \\
\text { réseau MADA (au } 1^{\text {er }} \text { mai 2018) }\end{array}$ & 9 & 6 & 8 & 15 & 13,1 & 820 \\
\hline $\begin{array}{l}\text { Proportion de localités faisant partie } \\
\text { du réseau MADA (au } 1^{\mathrm{er}} \text { mai } 2018 \text { ) }\end{array}$ & 69,2 & 75,0 & 40,0 & 37,5 & 64,9 & 74,8 \\
\hline
\end{tabular}

Source: Statistique Canada. Recensements de 1986 et de 2016. Compilation et calculs de l'auteur. 
$(42,4 \%)$. Pour ces raisons, le revenu moyen des familles se chiffre à 51584 \$, ce qui ne correspond qu'à 70,8\% de celui de la province. Leur précarité économique se double d'une marginalité géographique puisque ces petites localités se situent en moyenne à 40,5 kilomètres d'une ville, ce qui peut expliquer leur faible taux de migration pendulaire $(67 \%)$. Par ailleurs, neuf de ces 13 petites localités ont intégré le réseau Municipalité amie des aînés (MADA) 5 , ce qui constitue un signe du dynamisme des acteurs locaux (Ministère de la Famille et des Aînés, 2018).

À l'exception de Rivière-au-Tonnerre sise dans la MRC de Minganie, les localités dont la situation démographique est très précaire, au nombre de huit, se retrouvent toutes dans l'Est-duQuébec, soit quatre au Bas-Saint-Laurent et trois en Gaspésie. Leur taille démographique moyenne s'établit à 226 habitants. Ces milieux ont des caractéristiques qui s'apparentent en partie à celles des petites localités rurales présentant un niveau de précarité démographique extrêmement préoccupant. Elles souffrent en effet d'enclavement géographique, présentent un taux de chômage $(19,2 \%)$ nettement plus élevé que celui d'autres petites localités rurales et un faible taux d'activité $(43,1 \%)$. Enfin, malgré leur petite taille démographique, leur éloignement géographique et leurs problèmes économiques, les trois quarts des petites localités comprises au sein de cette catégorie ont intégré le réseau MADA.

On dénombre 20 petites localités dont la situation démographique est précaire. L'Est-duQuébec en englobe six, dont cinq au Bas-Saint-Laurent. La région Chaudière-Appalaches en comporte quatre. Deux se situent en Abitibi-Témiscamingue, sur la Côte-Nord ainsi que dans la MRC Charlevoix-Est. Les autres se dispersent au Saguenay-Lac-Saint-Jean, en Mauricie, en Estrie et dans le Nord-du-Québec. En l'espace de 30 ans, ces 20 localités, dont la taille démographique se chiffre à 278 habitants, ont perdu le tiers de leurs effectifs, ce qui représente une perte de 2785 personnes. Sur le plan économique, ces petites localités, bien que fragiles, tirent mieux leur épingle du jeu en comparaison à leurs homologues des deux groupes précédents. Cette catégorie se démarque, en effet, par un taux de chômage s'établissant à 9,7\%. Bien qu'elle soit inférieure à celle de l'ensemble des localités du Québec (68,5\%), la proportion du revenu provenant d'un emploi $(56,9 \%)$ des milieux appartenant à ce groupe demeure relativement élevée, du moins, si on le compare à celui des deux catégories précédentes.

Enfin, les 40 petites localités comprises dans la catégorie dont la situation démographique est plus ou moins précaire se caractérisent par un contexte socio-économique moins problématique que celui de leurs homologues des trois groupes précédents. À l'exception de la Gaspésie, toutes les régions périphériques du Québec en comportent au moins une avec une tendance à la concentration au Bas-Saint-Laurent, sur la Côte-Nord et en Abitibi-Témiscamingue. Plusieurs se retrouvent aussi dans la région Chaudière-Appalaches, celles des Hautes-Laurentides et en Estrie. Il s'agit du seul groupe à comporter des localités en croissance démographique, celles-ci étant au nombre de 22. Il n'en demeure pas moins que la population de ces petites localités a globalement régressé de 2,6 \% entre 1986 et 2016, ce qui traduit la très grande diversité démographique qui particularise ce groupe. La gérontocroissance caractérise ces petits milieux dont le nombre d'aînés a plus que doublé en 30 ans, alors que leur poids parmi l'ensemble de la population passait lui de

5. L'OMS définit une MADA comme une municipalité dont « les politiques, les services, les lieux et les structures soutiennent les personnes âgées en leur permettant de vieillir en restant actifs» (OMS, 2007: 5). 
$8,5 \%$ en 1986 à 24,2 \% en 2016. Sans être dynamique, l'économie locale semble moins affectée par le sous-emploi. Cela se reflète, en premier lieu, par un taux de chômage (10 \%) nettement inférieur à celui des petites localités dont la situation démographique est extrêmement $(27 \%)$ ou très précaire $(19,2 \%)$ et, deuxièmement, par une proportion du revenu provenant d'un emploi $(58,8 \%)$ supérieure à celle des petites localités dont le niveau de précarité démographique est soit extrêmement préoccupant $(42,4 \%)$ ou très précaire $(51,7 \%)$.

\section{CADRE D'INTERVENTION ET MESURES CORRECTRICES}

Suivant le paradigme de développement territorial exposé dans le cadre conceptuel, reproduit de manière schématique à la figure 3, les mesures à mettre en œuvre dans le but d'atténuer les effets du vieillissement tant « par le haut » que « par le bas » doivent agir sur deux leviers, c'est-à-dire sur le plan structurel (par la mise en place d'une politique d'aménagement du territoire et de croissance démographique) ainsi qu'en termes de renforcement des capacités. Parce qu'elle s'avère déterminante pour favoriser l'attractivité des territoires, la qualité de vie doit s'inscrire en filigrane dans ce cadre interventionnel (Godet et collab. 2018). Enfin, il s'avère impératif que les mesures à déployer s'inscrivent suivant une approche globale et qu'elles soient modulées en fonction de l'état de la situation démographique des petites localités.

\section{Mesures en lien avec le renforcement des capacités}

Le Québec fait figure de chef de file en matière de développement rural. De nombreux auteurs, dont Vachon $(2014,2011)$, Jean $(2012,2007)$ et Dugas (2003), pour ne citer que ceux-là, ont illustré, dans le cadre de leurs travaux, tout le chemin parcouru par le Québec afin de soutenir les acteurs locaux dans leurs efforts de développement. Bien que ce chemin ait été semé d'embûches, n’est-il pas légitime de se demander quelle serait la situation actuelle de nombreuses localités rurales en l'absence de l'appui des gouvernements provincial et fédéral ? En dépit de ses faiblesses, la défunte Politique nationale de la ruralité a exercé une contribution incontestable en favorisant le renforcement des capacités des acteurs locaux dans leur marche vers le développement. Considérée par l'Organisation de coopération et de développement économiques (OCDE) comme l'une des meilleures stratégies de développement rural ayant émané des pays développés, cette politique s'est échelonnée sur 13 ans, soit de 2002 à 2015 (OCDE, 2010). Par le biais des pactes ruraux, celle-ci a entraîné des effets bénéfiques en matière de développement local que ce soit en stimulant le leadership et l'entrepreneurship local, en favorisant l'empowerment, la participation citoyenne et la mobilisation sociale, en consolidant le sentiment d'appartenance et le capital socioterritorial, en promouvant le partenariat, en contribuant à la formation d'alliances et en établissant des formes inédites de gouvernance territoriale dans la perspective de mettre en ouvre un développement durable (Leblanc et collab. 2007).

C'est dans ce même esprit de renforcement des capacités qu'a été mis en place en 2005, à l'occasion du $18^{\mathrm{e}}$ Congrès mondial de gérontologie et de gériatrie, le réseau mondial «Villes amies des aînés » (VADA). L'initiative, qui vise à améliorer la qualité de vie des aînés au sein de leur milieu, a rapidement fait boule de neige pour s'étendre à des territoires géographiques plus restreints, si 
FIGURE 3

Le processus inhérent à la mise en œuvre d’une démarche de développement territorial

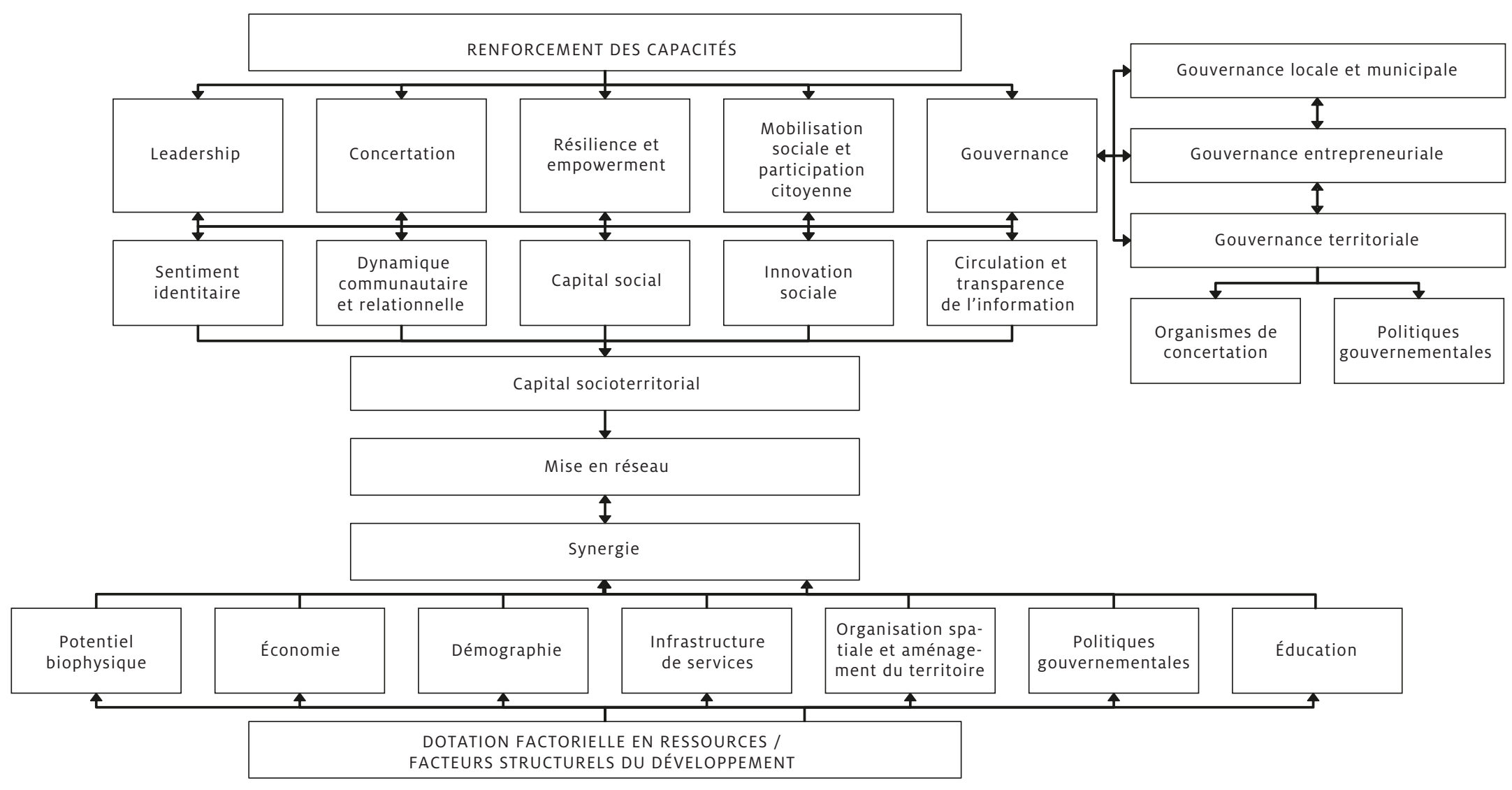

Majella Simard, Université de Moncton. 2018. 
bien que dans le cas du Canada, elle est mieux connue sous l'appellation MADA. L'originalité de la démarche tient essentiellement à deux aspects. Elle favorise, en premier lieu, le réseautage entre différents types d'acteurs provenant de différents milieux (communautaires, privés, publics, etc.). Par la suite, sa mise en œuvre repose sur la participation des aînés, ces derniers étant parties prenantes des différentes phases de son déploiement (Paris et collab. 2015; Liddle et collab. 2014; Paris et collab. 2013). En conséquence, en adhérant à la démarche MADA, les élus municipaux s'engagent à apporter les modifications nécessaires aux infrastructures et aux aménagements sis sur leur territoire en vue de mieux répondre aux besoins des aînés. De tels ajustements sont d'autant plus fondamentaux dans un contexte où la relation entre l'environnement (physique, naturel, humain et bâti) et les personnes âgées tend à s'intensifier, ces dernières souhaitant vieillir dans les milieux où elles ont vécu (Després et Lord, 2011; Milligan, 2009; Séguin et Apparicio, 2004). En date du $1^{\text {er }}$ mai 2018, le Québec comptait 820 MADA, ce qui représente les trois quarts des municipalités de la province ${ }^{6}$. De ce nombre, 563 appartenaient au monde rural, dont 131 (soit près du quart) correspondent à de petites localités. Cette seule constatation reflète bien la volonté des acteurs locaux à améliorer la qualité de vie des aînés ainsi que le dynamisme qui prévaut en milieu rural, et ce, même au sein des localités les plus fragiles. Cette dernière confirme aussi le fait que l'adaptation du cadre de vie des aînés est une réalité bien présente en milieu rural. À cet égard, qu'il soit permis de signaler le rôle d'avant-plan qu'exercent les entreprises d'économie sociale et les organismes de développement communautaire particulièrement dans les secteurs de l'habitation, des loisirs et du maintien à domicile (Lachapelle et Bourque, 2020; Broussy, 2014; Bourque et collab. 2011; Jetté et collab. 2011; Bouchard et collab. 2010).

Force est de reconnaître que les petites localités ne disposent pas des mêmes ressources humaines et financières que celles dont bénéficient les milieux à forte densité, les premières étant souvent confrontées au syndrome du TLM (Toujours Les Mêmes qui s'impliquent), ce qui, à bien des endroits, a pour effet de susciter la démobilisation des personnes les plus déterminées (Simard, 2018 ; Dugas, 1996). Dès lors, les premières se voient désavantagées par rapport aux secondes dans leurs efforts en matière d'aménagement, ce qui s'inscrit en porte à faux à un développement territorial durable (Jean, 2012; Angeon et collab. 2006). Il en est ainsi pour les aînés « qui ne sont pas toujours en mesure de peser autant sur les politiques publiques et les décisions qui les concernent » (Argoud, 2010 : 64). En conséquence, les initiatives déployées par les intervenants locaux et régionaux risquent de demeurer lettre morte si elles ne sont pas assorties d'interventions plus structurantes de la part de l'État en vue de les soutenir dans leurs efforts d'adaptation aux conditions de vie des aînés et à la rétention des jeunes, et ce, afin d'éviter une accentuation des inégalités sociales entre les municipalités (Séguin, 2012).

6. À titre comparatif, la Nouvelle-Écosse en comportait 13 (13,4 \% des municipalités), Terre-Neuve-etLabrador 41 (11 \%), le Nouveau-Brunswick, 22 (7,9\%) et l'Île-du-Prince-Édouard 3 (2,7\%). 


\section{Mesures structurelles en lien avec l’aménagement du territoire et redressement démo- graphique}

En dépit du dynamisme qui anime plusieurs acteurs locaux en ce qui a trait à l'amélioration de la qualité de vie de leurs résidents, ces mêmes acteurs s'avèrent impuissants à contrer les forces déstructurantes liées à la géographie et à la démographie, ce qui contribue à annihiler les effets de leurs interventions. Il s'ensuit que les différentes composantes inhérentes au renforcement des capacités ne peuvent être dissociées des éléments relatifs à la structure spatiale et à l'aménagement du territoire (Simard, 2016; Keating et collab. 2013). À ce chapitre, le Québec semble accuser un certain retard en comparaison avec plusieurs pays d'Europe dont les actions « ont favorisé l'émergence de nombreux dispositifs reposant sur le regroupement ou l'émergence de nouveaux acteurs locaux» (Gucher, 2014:121).

Conjugué à la dépopulation, le vieillissement entraîne incontestablement une déstructuration du peuplement, d'où la nécessité de déployer des actions musclées en matière d'aménagement du territoire, lesquelles doivent aller bien au-delà du cadre d'intervention proposé par le gouvernement du Québec dans sa Stratégie gouvernementale pour assurer l'occupation et la vitalité du territoire (Ministère des Affaires municipales et de l'Occupation du territoire, 2017). Par exemple, pour le Bas-Saint-Laurent où l'on retrouve 10 des 21 petites localités dont la situation démographique est très précaire, cette stratégie suggère de favoriser le développement social, de renforcer le sentiment d'appartenance et de promouvoir l'attractivité de la région. Or, ces trois mesures s'avèrent impuissantes pour inverser leur trajectoire démographique. Au chapitre de l'aménagement, deux actions apparaissent incontournables dans le but de renforcer la structure de peuplement de cette région, mais aussi de celles qui abritent plusieurs petites localités en difficulté sur le plan démographique, à savoir : le développement de nouveaux modes de prestation en matière de services de proximité et la création de pôles régionaux de développement.

Nous savons d'ores et déjà que les petites localités sont désavantagées en matière de services de proximité en raison des faibles effectifs à desservir, de la dispersion de leur population, de leur distance par rapport aux villes et, par conséquent, de leur difficulté à réaliser des économies d'échelle (Simard, 2003; Krout, 1998). En outre, bien que plusieurs de ces petits milieux soient intégrés au réseau MADA, rares sont ceux qui disposent d'un plan d'urbanisme ou d'un schéma d'aménagement qui évaluent les conséquences du vieillissement en matière d'aménagement et de développement territorial (Lord et Piché, 2018 ; Vachon, 2013). Par conséquent, c'est toute la question relative à la disponibilité et à l'accessibilité des services qu'il importe de redéfinir et d'adapter aux conditions ainsi qu'aux habitudes de vie tant des jeunes que des aînés (Bédard et Beaulieu, 2015).

Si l'échelon local n'est toujours pas le plus approprié ni le mieux adapté pour dispenser l'ensemble des services dont les aînés ont besoin, la création de pôles de services supralocaux, de schémas gérontologiques et le développement d'ententes intermunicipales semblent des avenues à privilégier particulièrement dans un contexte où les petites localités occupent généralement des espaces à faible densité d'une part et que plusieurs de celles-ci se situent dans des MRC dépourvues de villes de taille moyenne, voire en décroissance démographique de l'autre (Jean, 2007; 
Simard, 2005; Dugas, 2019, 2002, 1989). Ainsi, à l'exemple des contrats de pays ${ }^{7}$, ces pôles de services interviendraient à l'échelle du territoire (Blanchet, 2016, 2013; Gucher, 2014; Argoud, 2010 ; Ennuyer 2010 ; Grasset et Louargant, 2005). En outre, leur mandat et leurs affectations pourraient être intégrés aux orientations du schéma d'aménagement des MRC qui comporterait un volet consacré aux aînés comme c'est le cas en France avec les schémas gérontologiques départementaux (Argoud, 2007; Le Bouler, 2005). Les services qui y seraient dispensés (financier, poste, transport adapté, portage des repas, etc.) n'auraient pour limites que l'imagination des différents acteurs qui y seraient associés. Ces pôles contribueraient à consolider la structure de peuplement du milieu rural tout en favorisant la polarisation des activités économiques sur leur territoire respectif. Leur création pourrait aussi favoriser la rétention des personnes âgées au sein de leur milieu en plus de renforcer le potentiel de développement de celui-ci (Dugas, 2002). C'est donc dans une perspective multiscalaire qui prend en compte la globalité du processus du vieillissement qu'une politique d'aménagement du territoire doit se déployer (Broussy, 2014; Argoud et collab. 2006).

Dans bien des cas, les municipalités orientent leurs actions vers la préservation de leurs acquis au lieu de préconiser la diversification de l'offre de services. Pour cette raison, les ententes intermunicipales de services sont relativement peu développées en milieu rural. Lorsqu'elles existent, celles-ci se limitent trop souvent à la protection contre les incendies, au ramassage des ordures ou au déneigement. Dès lors, ces ententes pourraient contribuer à élargir la brochette de services offerts en milieu rural en considérant par exemple l'offre en matière de transport, le logement et les loisirs (Negron-Poblète et collab. 2012). Bref, à l'instar des villes qui ont développé une culture d'innovation dans la gestion et la planification des services, les milieux ruraux, en particulier les petites localités, sont conviés à faire preuve d'audace en cette matière (Venne, 2018).

Dans la mesure où de nombreuses petites localités sont confrontées simultanément au vieillissement accéléré de leur population, tant "par le haut » que " par le bas », ainsi qu'à l'émigration des jeunes, ces derniers doivent également retenir l'attention des instances gouvernementales dans l'élaboration d'une stratégie d'aménagement du territoire (Morin et Van Den Bussche, 2018). Plusieurs organismes ont mis en place différents plans d'action en vue de retenir les jeunes actifs et d'accroître l'attractivité des milieux ruraux. Pensons notamment aux Carrefours Jeunesse Emploi, à Place aux Jeunes, à Emploi-Québec, à la Stratégie jeunesse de certaines Sociétés d'aide au développement des collectivités (SADC) ainsi qu'aux nombreuses initiatives (embauche d'agents de migration et communautaires, bourse d'attractivité, etc.) mises en place par les municipalités et les MRC. Il importe non seulement de renforcer leurs actions, mais aussi de les coupler à des inci-

7. En France, le «pays » est « une organisation locale liée à la politique d'aménagement du territoire » (Doré, 2011: 89). Couvrant la presque totalité de l'espace national et financée par la région, cette structure sert de cadre aux acteurs locaux qui décident de se regrouper pour unir leurs forces en vue d'élaborer des projets en lien avec l'aménagement du territoire notamment afin de favoriser un meilleur accès aux services. «Les contrats sont signés généralement au minimum entre la Région et la structure porteuse du Pays (syndicat mixte, association, GIP - Groupement d'intérêt public Aménagement du territoire) » (Doré, 2014: 165). Ces contrats s'inscrivent « à la fois dans un objectif de coordination, de meilleure couverture du territoire régional et de négociation avec les interlocuteurs locaux » (Doré, $2014: 161)$. 
tatifs financiers destinés aux jeunes adultes et aux familles avec enfants afin de bonifier leurs résultats. La création d'emplois, la diversification de l'économie et la consolidation de l'infrastructure de services locaux apparaissent des conditions incontournables afin d'inciter les jeunes à demeurer au sein de leur milieu et d'en attirer de nouveaux.

Une stratégie d'aménagement du territoire favorisera une meilleure structuration de l'espace à la condition qu'elle soit couplée à une politique misant sur la conciliation travail/famille et sur la solidarité intergénérationnelle. Par exemple, on peut penser à des crédits d'impôt aux familles (déterminés en fonction du nombre d'enfants), à la bonification du programme d'allocations familiales, à la libéralisation de temps pour la famille (congés parentaux, flexibilité des horaires de travail, etc.), à la flexibilisation du travail ainsi qu'à l'établissement de rapports plus égalitaires entre hommes et femmes, le but étant de favoriser la participation au marché du travail des femmes qui souhaitent avoir des enfants (Dubreuil et Marois, 2011; Henripin, 2004).

Un meilleur contrôle des mouvements migratoires interrégionaux apparaît aussi une mesure essentielle particulièrement dans le cas du Bas-Saint-Laurent. En effet, avec celles de Montréal et du Nord-du-Québec, cette région est l'une des seules à maintenir, d'année en année, un bilan migratoire négatif. Abritant plusieurs petites localités sujettes au vieillissement, les MRC de La Matapédia et de Témiscouata sont particulièrement touchées par ce phénomène (Institut de la Statistique du Québec, 2018). Son atténuation passe par des mesures destinées à accroître l'attractivité du territoire telles que des congés de taxes, des allocations visant à encourager l'arrivée de jeunes actifs, de néo-ruraux ou de retraités, la mise en place de budgets participatifs et la création d'emplois au sein des petites localités dont la situation démographique est considérée comme étant extrêmement préoccupante. Bien qu'intéressantes, d'autres actions, comme la stimulation de l'immigration, apparaissent, par ailleurs, plus difficilement applicables particulièrement en milieu rural (Sauvé, 2016; Dubreuil et Marois, 2011).

\section{CONCLUSION}

Les petites localités se distinguent par un vieillissement prononcé de leur population, leur tendance à la décroissance démographique ainsi que par l'exode d'une partie de leurs effectifs notamment des jeunes, à la recherche de meilleures perspectives d'emploi, mais aussi des personnes âgées désireuses de profiter de la variété des services offerts en milieu urbain. Ces trois phénomènes se manifestent suivant un rythme et des intensités variables selon les endroits. Si les petites localités rurales sises près des villes ou à forte vocation touristique sont affectées par le vieillissement " par le haut ", elles sont moins touchées par la diminution de leur proportion de jeunes, et donc, par la décroissance de leurs effectifs, en comparaison à leurs homologues qui ne profitent pas de la proximité d'une agglomération urbaine et dont la population se disperse sur un vaste territoire. C'est le cas des petites localités situées dans la plupart des régions périphériques du Québec, comme au BasSaint-Laurent, en Gaspésie, sur la Côte-Nord, en Abitibi-Témiscamingue et dans le Nord-duQuébec, mais aussi de celles dites « intermédiaires » comme en Outaouais ou dans la région Chaudière-Appalaches. Plusieurs des petites localités sises dans ces régions présentent une situation démographique extrêmement préoccupante qui risque de compromettre très sérieusement leur avenir. 
Au cours des dernières années, le gouvernement du Québec a mis en place tout un arsenal de mesures (programmes de soutien à domicile, aide financière pour les services d'aide-domestique, allocation-logement, habitations à loyer modique pour les aînés à faible revenu, transport adapté, etc.) et de politiques destinées à renforcer la capacité des acteurs à agir sur le développement de leur territoire et à améliorer la qualité de vie des individus qui l'occupe en particulier des personnes âgées. Toutefois, ces mesures ont été développées sans égard aux diverses caractéristiques de la structure spatiale (comme la taille démographique des municipalités, leur distance par rapport à la ville, le degré de dispersion de la population, etc.), aux considérations socio-économiques des milieux concernés (nature de l'économie, niveau de revenu, etc.) ou à l'intensité de leur vieillissement et/ou de leur gérontocroissance ou de leur décroissance, ce qui en réduit considérablement la portée. Or, il s'avère essentiel que les différents intervenants œuvrant dans le domaine de l'aménagement du territoire, de concert avec l'État, déploient, de façon concertée, des actions qui prennent en compte ces différents paramètres de manière à favoriser un développement territorial intégré. Il s'avère aussi impératif que les actions des intervenants locaux s’insèrent dans une approche plus globale de développement rural, et ce, d'autant plus que les petites localités reçoivent habituellement peu d'attention auprès des diverses instances gouvernementales en raison de leur faible poids politique. À ce chapitre, une éventuelle politique d'aménagement du territoire devrait prendre en compte les changements des trajectoires démographiques, tant aux échelons national, régional que local, en considérant la situation particulière des petites localités en fonction de l'intensité de leur vieillissement et de leur situation géographique (en particulier de leur distance par rapport aux villes) et économique. De manière plus spécifique, les actions à mettre en œuvre doivent être envisagées dans l'optique de favoriser le rééquilibrage de la structure de peuplement et de promouvoir la mise en place d'une politique démographique tournée vers la stimulation de la fécondité, une meilleure régulation des flux migratoires et des mesures d'attractivité à destination des jeunes. Dans un contexte où les trajectoires de mobilité des individus sont de plus en plus à géométrie variable, ces actions doivent s’inscrire dans un cadre multiscalaire de façon à favoriser l'égalité des chances non seulement des personnes âgées, mais aussi des jeunes, et ce, afin d'améliorer leur qualité de vie et de promouvoir une occupation dynamique du territoire.

\section{BIBLIOGRAPHIE}

ANGEON, V., P. Caron et S. Lardon. 2006. «Des liens sociaux à la construction d'un développement territorial durable : quel rôle de la proximité dans ce processus? ", Développement durable et territoires.

ARGOUD, D. 2010. « Approche historique des dispositifs gérontologiques en France », Gérontologie et Société, 33, 132: 103-110.

ARGOUD, D. 2007. "La décentralisation de la politique vieillesse. Vers une accentuation des inégalités?", Gérontologie et société, 123: 201-212.

ARGOUD, D., G. BESSAC et P.-H. DAURE. 2006. «Territoires et vieillissement ", Les Annales de la recherche urbaine, $100: 2-32$.

BEAUDET, G. et J.-P. MELOCHE. 2012. «L'aménagement du territoire au Québec : le parent pauvre des sciences régionales », Revue d'économie régionale et urbaine, $4:$ 691-716. 
BÉDARD, M.-È et M. BEAULIEU. 2015. «La défense des droits des aînés au Québec : protection et empowerment ", Le Sociographe, $50: 33-42$.

BISSON, L. 2013. Le renforcement des capacités de développement des collectivités rurales : l'expérience des SADC au Québec. Rimouski, Université du Québec à Rimouski, 92 p.

BLANCHET, M. 2013. "Le vieillissement des campagnes : éléments d'introduction démographique et géographique », Gérontologie et société, 36, 146 : 21-38.

BLANCHET, M. 2016. «Regards croisés d'actions gérontologiques territorialisées sur la loi hôpitalpatients-santé et territoires (hpst) », Annales de géographie, 708 : 170-192.

BLASQUIET-REVOL, H., C. DABAU, M.-A. LENAIN, J.-F. MANDY, M. GLENAT, C. GAUDEMER et M. KOCH-MATHIAN. 2011. "Les retraités : acteurs de la création d'activités », Gérontologie et Société, $138:$ 51-65.

BONTRON, J.-C. et S. CABANIS. 1993. Essai de typologie socio-économique des cantons français. Paris, Société d'études géographiques, économiques et sociologiques appliquées, 18 p.

BOUCHARD, M.-J., W. FROHN et R. MORIN. 2010. «Le logement communautaire au Québec: apport et limites d'innovation sociale », Lien social et Politiques, 63: 93-193.

BOURQUE, D., R. LACHAPELLE, D. MALTAIS, S. SAVARD et M. TREMBLAY. 2011. "Création des CSSS et impacts sur les rapports avec les organismes communautaires et d'économie sociale dans le programme Perte d'autonomie liée au vieillissement », Service social, 57, $1:$ 80-98.

BROUSSY, L. 2014. Dix mesures pour adapter la société française au vieillissement. Paris, Éditions Dunod, $228 \mathrm{p}$.

CAPELLO, R. 2014. "Proximity and regional innovation », dans A. TORRE et F. WALLET (dir.), Regional development and proximity relations, Cheltenham, UK et Northampton, Edward Elgar : 163-194.

COMMISSION MONDIALE SUR L'ENVIRONNEMENT ET LE DÉVELOPPEMENT. 1988. Notre avenir à tous. Montréal, Éditions du Fleuve, $454 \mathrm{p}$.

DAVIS, C. A. 2008. Le renforcement des capacités : l'habilitation des gens et des institutions. Rapport annuel. New York, Programme des Nations unies sur le développement, 39 p.

DAVAZIES, L. 2009. "Il y a économie résidentielle et économie résidentielle », Pour, 100 : 50-52.

DESPRÉS, C. et S. LORD. 2011. «Vieillir en banlieue nord-américaine. Le rapport à la ville des personnes âgées », Gérontologie et société, 34, 136:189-204.

DIALGA, I. et T.-H.-G. LE. 2014. Développement d'indices composites et politiques publiques : interactions, portée et limites méthodologiques. Nantes, Laboratoire d'économie et de management Nantes-Atlantique- IAE, $38 \mathrm{p}$.

DORÉ, G. 2014. "La contractualisation territoriale des conseils régionaux », Revue d'économie régionale et urbaine, $1: 157-172$.

DORÉ, G. 2011. « Le pays, un singulier pluriel », Pour, 209-210 : 89-102.

DUBREUIL, B. et G. MAROIS. 2011. Le remède imaginaire : pourquoi l'immigration ne sauvera pas le Québec. Montréal, Éditions du Boréal, 315 p.

DUGAS, C. 1989. "L'aménagement dans un espace à population dispersée », Revue québécoise d'urbanisme, 9, 3:9-23.

DUGAS, C. 1996. L'espace rural canadien. Sainte-Foy, Presses de l'Université du Québec, 217 p. 
DUGAS, C. 2019. «Le renforcement des centres de services : un moyen d'atténuation des disparités territoriales ", dans M.-U. PROULX et M.-C. Prémont (dir.), La politique territoriale au Québec. 50 ans d'audace, d'hésitations et d'impuissance, Québec, Presses de l'Université du Québec: 273-301.

DUGAS, C. 2003. "Les politiques de développement régional au Canada depuis 1960 : éléments de bilan », Conférence présentée à l'Université du Québec à Rimouski lors du congrès annuel de l'ACFAS, disponible à la page : http ://classiques.uqac.ca/desintegration/dugas_clermont/ pol_devel_regional_canada/pol_devel.html.

DUGAS, C. 1990. "Pour une politique québécoise de l'aménagement du territoire », L'Action nationale, $80,8: 1100-1115$.

DUGAS, C. 2002. "Une stratégie de renforcement des petits centres ruraux », Revue Organisations et Territoires, 11, $1: 11-16$.

DUMONT, G.-F. 2012. Diagnostic et gouvernance des territoires. Concepts, méthode, application. Paris, Éditions Armand Colin, 299 p.

DUMONT, G.-F. 2006. Les territoires face au vieillissement en France et en Europe. Géographie, Politique, Prospective. Paris, Éditions Ellipse, 416 p.

DUMONT, G.-F. 2016. "Vieillissement de la population : quatre types de dynamique territoriale », Revue politique et parlementaire, 118, $1081:$ 51-59.

ENNUYER, B. 2010. «Territoire gérontologique? Quels sens donner à cette locution », Gérontologie et Société, 33, 132:165-170.

ESTĖBE, P., Y. JANVIER, S. TIEVANT et L. DAVEZIES. 2007. L'économie résidentielle et le développement local : conséquences ou levier? Acadie-Paris, Groupe Relex, 116 p.

FORTIN, M.-J. et G. CHIASSON. 2017. Pour un Québec fort de toutes ses régions. Se donner les moyens de nos ambitions collectives. Rimouski, Centre de recherche sur le développement territorial, $16 \mathrm{p}$.

GODET, M., B. JARRY et D. CHABAUD. 2018. «Innovation et territoires : les limites de la " métropolisation » en France », Population et Avenir, $738:$ 4-7.

GRASSET, É. et S. LOUARGANT. 2005. Une plate-forme de services en Sud Ardèche, une opportunité de développement territorial. Lyon et Grenoble, Centre national de recherche scientifique, $93 \mathrm{p}$.

GUCHER, C. 2014. "Vieillissement dans les espaces ruraux en France et « effets milieu » : enjeux humains et territoriaux des mutations démographiques », Cahiers québécois de démographie, 43, $1: 103-132$.

GUMUCHIAN, H. 1990. À la périphérie de la périphérie : l'espace rural et le concept de fragilité en Abitibi. Montréal, Université de Montréal, 66 p.

HENRIPIN, J. 2004. Pour une politique de population. Montréal, Éditons Varia, 121 p.

HOUÉE, P. 1996. Les politiques de développement rural. Des années de croissance au temps des incertitudes, $2 e$ édition. Paris, Éditions Économica, $321 \mathrm{p}$.

INSTITUT DE LA STATISTIQUE DU QUÉBEC. 2018. Solde migratoire et taux net de migration interne, MRC du Québec classées par région administrative, disponible à la page : http ://www. stat.gouv.qc.ca/statistiques/population-demographie/migration/internes/migration-int-mrcsolde-taux.html 
JEAN, B. 2012. «Les territoires ruraux au Québec : vers un modèle de développement territorial durable », Revue d'économie régionale et urbaine, 4:649-671.

JEAN, B. 2007. «Réussir le développement des milieux ruraux de faible densité : les enseignements de l'expérience québécoise ", dans L. BARTHE, F. CAVAILLE, C. EYCHENNE et J. PILLEBOUC (dir.), Habiter et vivre dans les campagnes de faible densité, Clermont-Ferrand, Presses universitaires Blaise Pascal : 171-198.

JETTÉ, C., Y. VAILLANCOURT et J.-V. BERGERON-GAUDIN. 2011. «L'économie plurielle dans les services à domicile au Canada : une comparaison des modes de régulation entre le Québec et l'Ontario », Lien social et Politiques, $66:$ 155-175.

KEATING, N., J. EALES, et J.E PHILLIPS. 2013. « Age-Friendly Rural Communities. Conceptualizing « best-fit » ", Revue canadienne du vieillissement, 32, 4:319-332.

KROUT, J. A. 1998. "Services and Service Delivery in Rural environments », dans R.T. COWARD et J.A. KROUT (dir.), Aging in Rural Settings : Life, Circumstances and Distinctive Futures, New York, Springer Publishing Company: 247-266.

LACHAPELLE, R. et D. BOURQUE. 2020. Intervenir en développement des territoires. Québec, Presses de l'Université du Québec, 148 p.

LEBLANC, P., B. JEAN, D. LAFONTAINE et M. MARCEAU. 2007. Le modèle et les indicateurs de suivi et d'évaluation du dispositif du pacte rural. Rimouski, Centre de recherche sur le développement territorial, $103 \mathrm{p}$.

LE BOULER, S. 2005. "Personnes âgées dépendantes : le temps de la planification », Les Tribunes de la santé, $7: 49-58$.

LIDDLE, J., T. SCHARF, B. BARTLAM, M. BERNARD et J. SIM. 2014. " Exploring the agefriendliness of purpose-built retirement communities : evidence from England », Ageing and Society, 34, 9 : 1601-1629.

LORD, S. et D. PICHÉ. 2018. « Un enjeu structurant pour nos milieux de vie », dans S. LORD et D. PICHÉ (dir.), Vieillissement et aménagement. Perspectives plurielles, Montréal, Presses de l'Université de Montréal : 9-22.

MERLIN, P. 1988. Géographie de l'aménagement. Paris, Presses universitaires de France, 334 p.

MILLIGAN, C. 2009. There's No Place Like Home : Place and Care in an Ageing Society. Burlington, Ashgate Publishing, $188 \mathrm{p}$.

MINISTÈRE DE LA FAMILLE ET DES AÎNÉS. 2018. Liste des municipalités amies des aînés, disponible à la page : https ://www.mfa.gouv.qc.ca/fr/aines/mada/liste-mada/Pages/index.aspx

MINISTĖRE DES AFFAIRES MUNICIPALES ET DE L'OCCUPATION DU TERRITOIRE. 2017. Stratégie gouvernementale pour assurer la vitalité territoires 2018-2022. Les régions aux commandes. Québec, Gouvernement du Québec, 108 p.

MORIN, D. et C. VAN DEN BUSSCHE. 2018. «Vieillissement et transformation des milieux de vie à Québec », dans S. LORD et D. PICHÉ (dir.), Vieillissement et aménagement. Perspectives plurielles, Montréal, Presses de l'Université de Montréal : 55-77.

NEGRON-POBLETE, P., A.-M. SÉGUIN et P. APPARICIO. 2012. "Accessibilité à pied pour les résidents âgés du Vieux-Longueuil. Des problèmes à l'horizon », dans P. NEGRON-POBLÈTE et A.-M. SÉGUIN (dir.), Vieillissement et enjeux d'aménagement. Regards à différentes échelles, Québec, Presses de l'Université du Québec, 2012 : 155-178. 
ORGANISATION DE COOPÉRATION ET DE DÉVELOPPEMENT ÉCONOMIQUES. 2010. Examens de l'OCDE des politiques rurales, Québec, Canada. Paris, Éditions de l'OCDE, 343 p.

ORGANISATION DES NATIONS UNIES. 2003. Déclaration politique et Plan d'action international de Madrid sur le vieillissement. New York, Organisation des Nations unies, $53 \mathrm{p}$.

ORGANISATION DES NATIONS UNIES. 2017. Les personnes âgées, disponible à la page : http :// www.un.org/fr/sections/issues-depth/ageing/index.html

ORGANISATION MONDIALE DE LA SANTÉ. 2007. Guide mondial des villes-amies des aînés. Vieillissement et qualité de vie, santé familiale et communautaire. Genève, $78 \mathrm{p}$.

PARIS, M., S. GARON, et M. BEAULIEU. 2015. "La participation sociale dans quatre projets pilotes Municipalités amies des aînés : la vie sociale et récréative au cour du processus de planification et d'implantation ». ACFAS, Rimouski, Canada.

PARIS, M., S., GARON, P.-M. CHAPON, A. LALIBERTÉ et A. VEIL. 2013. " Le programme «Municipalités amies des aînés au Québec », un modèle pour la France », Retraite et société, 64 : 181-189.

POLĖSE, M., R. SHEAMUR et L. TERRAL. 2015. Économie urbaine et régionale. Géographie économique et dynamique des territoires. Paris, Éditions Économica, $416 \mathrm{p}$.

RICAN, S., É. JOUGLA, Z. VAILLANT et G. SALEM. 2013. "Les inégalités territoriales de santé », dans É. LAURENT (dir.), Vers l'égalité des territoires. Dynamiques, mesures, politiques, Paris, La Documentation française : 113- 129.

SAUVÉ, M.-R. 2016. "L'immigration est un « remède imaginaire » au déclin démographique », Le Devoir, disponible à la page : https ://www.ledevoir.com/non-classe/470237/l-immigration-estun-remede-imaginaire-au-declin-demographique.

SEGESSMANN, A. et O. CREVOISIER. 2013. "L'économie résidentielle par les emplois », Revue d'économie régionale et urbaine, $4: 705-735$.

SÉGUIN, A.-M. 2012. "Le réaménagement des villes pour nos aînés. Une opportunité à saisir », dans P. NEGRON-POBLÈTE et A.-M. SÉGUIN (dir.), Vieillissement et enjeux d'aménagement. Regards à différentes échelles, Québec, Presses de l'Université du Québec : 213-218.

SÉGUIN, A.-M. et P. APPARICIO. 2004. "Évolution de la distribution spatiale de la population âgée dans la région métropolitaine entre 1981 et 2001 : constat et enjeux pour les municipalités », Revue canadienne de science régionale, 27, $1: 79-98$.

SIMARD, M. 2003. La fragilité de l'espace rural québécois. Le cas des petites localités du Bas-SaintLaurent : enjeux et perspectives d'avenir. Rimouski, Université du Québec à Rimouski, 968 p.

SIMARD, M. 2016a. La contribution de l'économie sociale au développement des milieux ruraux et urbains du Bas-Saint-Laurent. Perception des gestionnaires d'entreprises. Rimouski, Éditions du GRIDEQ, $187 \mathrm{p}$.

SIMARD, M. 2015a. "La géographie des disparités économiques au sein des localités du Canada atlantique : une étude comparative au cours de la période 1981-2006», Port Acadie, 27 : 83-117.

SIMARD, M. 2005. «Les espaces à faible densité : un défi au développement des milieux ruraux québécois », Revue canadienne des sciences régionales, 28, 1:303-329.

SIMARD, M. 2018. Le JAL : un demi-siècle de luttes et débats. Bilan et perspectives nouvelles d'une expérience de développement communautaire en milieu rural fragile. Rimouski, Éditions du GRIDEQ, $236 \mathrm{p}$. 
SIMARD, M. 2016. «Les petites localités rurales de l'Atlantique : une analyse sous l'angle de la fragilité et de la fragilisation territoriale ", Revue de l'Université de Moncton, 47, 1: 101-135.

SIMARD, M. 2015b. "Quelques jalons en vue de l'élaboration d'une politique territoriale du vieillissement au Nouveau-Brunswick », Vie et vieillissement, 12, 3:33-41.

STATISTIQUE CANADA. 1986. Recensement de 1986. Ottawa, Division de la diffusion informatique des données, CD-ROM.

STATISTIQUE CANADA. 2016. Recensement de la population, disponible à la page : http :// www12.statcan.gc.ca/census-recensement/2016/dp-pd/dt-td/Rp-fra.cfm? LANG=F\&APATH=3 $\& D E T A I L=0 \& D I M=0 \& F L=A \& F R E E=0 \& G C=0 \& G I D=0 \& G K=0 \& G R P=1 \& P I D=109648 \& P R I$ $\mathrm{D}=10 \& \mathrm{PTYPE}=109445 \& \mathrm{~S}=0 \& \mathrm{SHOWALL}=0 \& \mathrm{SUB}=0 \& \mathrm{Temporal}=2016 \& \mathrm{THEME}=117 \& \mathrm{VID}=0-$ $\& \mathrm{VNAMEE}=\& \mathrm{VNAMEF}=$

VACHON, B. 2011. La passion du rural. Quarante ans d'écrits, de paroles et d'actions pour que vive le Québec rural. Tome 1. Trois-Pistoles, Éditions Trois-Pistoles, 524 p.

VACHON, B. 2014. La passion du rural. Quarante ans d'écrits, de paroles et d'actions pour que vive le Québec rural. Tome 2. Évolution récente du Québec rural, 1961-2014 : de l'exode au puisant désir de campagne. Trois-Pistoles, Éditions Trois-Pistoles, 535 p.

VACHON, B. 2013. " Les enjeux de la ruralité en 2013 ", Urbanité :18-19.

VENNE, J.-F. 2018. «Des villes inventives », Le Devoir, disponible à la page : https ://www.ledevoir.com/politique/quebec/527250/des-villes-inventives. 